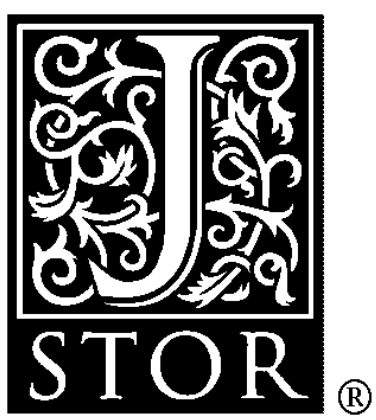

Optimal Multiserver Stochastic Scheduling of Two Interconnected Priority Queues Author(s): Dimitrios G. Pandelis and Demosthenis Teneketzis

Source: Advances in Applied Probability, Vol. 26, No. 1, (Mar., 1994), pp. 258-279

Published by: Applied Probability Trust

Stable URL: http://www.jstor.org/stable/1427590

Accessed: 04/04/2008 12:05

Your use of the JSTOR archive indicates your acceptance of JSTOR's Terms and Conditions of Use, available at http://dv1www56.jstor.org:6085/page/info/about/policies/terms.jsp. JSTOR's Terms and Conditions of Use provides, in part, that unless you have obtained prior permission, you may not download an entire issue of a journal or multiple copies of articles, and you may use content in the JSTOR archive only for your personal, non-commercial use.

Please contact the publisher regarding any further use of this work. Publisher contact information may be obtained at http://www.jstor.org/action/showPublisher?publisherCode=apt.

Each copy of any part of a JSTOR transmission must contain the same copyright notice that appears on the screen or printed page of such transmission.

JSTOR is a not-for-profit organization founded in 1995 to build trusted digital archives for scholarship. We enable the scholarly community to preserve their work and the materials they rely upon, and to build a common research platform that promotes the discovery and use of these resources. For more information about JSTOR, please contact support@jstor.org. 


\title{
OPTIMAL MULTISERVER STOCHASTIC SCHEDULING OF TWO INTERCONNECTED PRIORITY QUEUES
}

\author{
DIMITRIOS G. PANDELIS AND \\ DEMOSTHENIS TENEKETZIS * University of Michigan
}

\begin{abstract}
A number of jobs on two interconnected queues are to be processed by $m$ identical servers. The servers operate in parallel, so that every server can process any job. Jobs in queue $i, i=1,2$, incur an instantaneous holding cost $C_{i}$ during the time they remain in the system. The service time for jobs in queue $i$, denoted by $X_{i}$, is a random variable with a general distribution. The interconnection process is independent of the service process. We establish sufficient conditions on the service times, the holding costs and the interconnection process under which the non-preemptive scheduling strategy that gives priority to queue 1 minimizes the total expected $\alpha$-discounted cost. We call this strategy P1. We present counterexamples showing that if any of the sufficient conditions is not satisfied P1 may not be optimal, and that the optimal policy for the single-server problem is not necessarily optimal for the multiserver problem.
\end{abstract}

MULTISERVER SCHEDULING; INTERCONNECTED QUEUES; STOCHASTIC ORDERING

AMS 1991 SUBJECT CLASSIFICATION: PRIMARY 93E05

SECONDARY 90B35

\section{Introduction}

We consider two interconnected queues that are served by $m$ identical servers. The servers operate in parallel, so that every server can process any job. The queues are interconnected in the following way: jobs that complete service in queue 1 join queue 2 with probability $p$, and leave the system with probability $1-p$. Jobs that complete service in queue 2 leave the system. We assume that the interconnection process is independent of the service process. Jobs in queue $i, i=1,2$, incur an instantaneous holding cost $C_{i}$ during the time they remain in the system. The service time for jobs in queue $i, i=1,2$, denoted by $X_{i}$, is a random variable with a general distribution; we assume that the service times are independent and $X_{1}$ has non-decreasing likelihood ratio. There are no arrivals, so we are concerned with the

Received 27 July 1992; revision received 21 December 1992.

* Postal address for both authors: Department of Electrical Engineering and Computer Science, University of Michigan, Ann Arbor, MI 48109-2122, USA. 
scheduling of jobs initially present in the system. We seek to determine a non-preemptive scheduling strategy $\pi$ that minimizes the total expected $\alpha$ discounted holding cost given by

$$
\begin{gathered}
J^{\pi}\left(y_{1}, y_{2}\right)=\boldsymbol{E}\left\{\sum_{j=1}^{y_{1}}\left[\int_{0}^{t_{j}} C_{1} \exp (-\alpha t) d t+1_{j} \int_{t_{j}}^{t_{1}} C_{2} \exp (-\alpha t) d t\right]\right. \\
\left.+\sum_{j=1}^{y_{2}} \int_{0}^{s_{j}} C_{2} \exp (-\alpha t) d t\right\},
\end{gathered}
$$

where

(1.2) $y_{i}, i=1,2$, is the length of queue $i$ at time 0 ;

(1.3) $t_{j}, j=1,2, \cdots, y_{1}$, is the time the $j$ th job in queue 1 is completed,

(1.4) $l_{j}, j=1,2, \cdots, y_{1}$, is the time the $j$ th job initially in queue 1 is completed in queue $2\left(l_{j}=t_{j}\right.$ if that job does not join queue 2 after being processed in queue 1),

(1.5) $s_{j}, j=1,2, \cdots, y_{2}$, is the time the $j$ th job in queue 2 is completed;

(1.6) $1_{j}, j=1,2, \cdots, y_{1}$, is the indicator random variable taking the value of 1 if the $j$ th job completed in queue 1 joins queue 2 , and taking the value of 0 otherwise.

To the best of our knowledge, this is the first attempt to determine optimal strategies for multiserver scheduling problems in interconnected queues. Most of the work on optimization in multiserver scheduling up till now has concentrated on parallel queues, where the jobs have equal priorities, that is, all jobs incur the same cost or reward. Two strategies appear almost exclusively in the literature achieving optimality for various performance criteria, SEPT (the strategy that processes jobs in increasing order of expected service time) and LEPT (the strategy that processes jobs in decreasing order of expected service time). For results when the service times are exponentially distributed see the work of Glazebrook and Nash (1976), Glazebrook (1979), Weiss and Pinedo (1980), Bruno et al. (1981), Van Der Heyden (1981), Weiss (1982), Coffman et al. (1987), and Lehtonen (1988). When the service times have a monotone hazard rate, see the work of Weber (1980), (1982). Weber et al. (1986), whose approach we follow in this paper, considered the problem of scheduling jobs with service times that are stochastically ordered, when an instantaneous reward which is a convex decreasing function of time is earned upon each service completion. They proved that the strategy SEPT maximizes the total expected reward. The results on scheduling of jobs with different priorities are 
relatively few. For jobs with exponential service times and two servers Ross (1983) showed that, when the LEPT strategy and the $c \mu$-rule result in the same order, the $c \mu$-rule minimizes the expected weighted sum of completion times. Kampke (1987), (1989) extended the result to $m$ servers given that, if $c_{i} \geqq c_{j}$, then $c_{i} \mu_{i} \geqq c_{j} \mu_{j}$. Chang et al. (1991) showed that for exponential service times and two classes of jobs the optimal policy is of threshold type. Asymptotic results on multiserver scheduling of jobs with priorities can be found in Whittle (1988), Weber and Weiss (1990), and Agrawal et al. (1990) in the context of multi-armed bandits. Finally, $\mathrm{Xu}$ et al. (1990) studied the scheduling of multipriority jobs on two heterogeneous servers. They proved that for each priority class the non-preemptive scheduling strategy that minimizes expected flowtime is of the threshold type. In this paper we establish sufficient conditions on the service times and the holding costs under which the non-preemptive scheduling strategy that gives priority to queue 1 minimizes the total expected $\alpha$-discounted holding cost. We call this strategy P1.

The rest of the paper is organized as follows. In Section 2 we prove the optimality of strategy P1. In Section 3 we provide counterexamples illustrating the following points: (i) if any of the sufficient conditions of Theorem 1 is relaxed the optimality of P1 is not guaranteed; (ii) when the conditions of Theorem 1 are satisfied but the queues are interconnected in the opposite way strategy P1 may not be optimal; (iii) the conditions of Theorem 1 are sufficient but not necessary for the optimality of P1; and (iv) the index rule that describes the optimal policy in the single-server problem is suboptimal in the multiserver problem.

\section{Sufficient conditions for the optimality of strategy P1}

The discounted holding cost problem stated in the introduction can be studied in terms of the following equivalent reward problem. A job completed in queue $i$, $i=1,2$, at time $t$ incurs a reward $g_{i}(t)$, where $g_{1}(t)=\left(C_{1}-p C_{2}\right) \exp (-\alpha t)$, $g_{2}(t)=C_{2} \exp (-\alpha t)$. The expected reward due to a strategy $\pi$ is then

$$
R^{\pi}\left(y_{1}, y_{2}\right)=\boldsymbol{E}\left\{\sum_{j=1}^{y_{1}}\left[\left(C_{1}-p C_{2}\right) \exp \left(-\alpha t_{j}\right)+1_{j} C_{2} \exp \left(-\alpha l_{j}\right)\right]+\sum_{j=1}^{y_{2}} C_{2} \exp \left(-\alpha s_{j}\right)\right\},
$$

where $y_{i}, i=1,2, t_{j}, j=1,2, \cdots, y_{1}, l_{j}, j=1,2, \cdots, y_{1}, s_{j}, j=1,2, \cdots, y_{2}$, and $1_{j}$, $j=1,2, \cdots, y_{1}$, have been defined by Equations (1.2)-(1.6). We show that the policy that minimizes the total expected $\alpha$-discounted holding cost maximizes the 
total expected reward. This can be seen as follows:

$$
\begin{aligned}
J^{\pi}\left(y_{1}, y_{2}\right)= & \boldsymbol{E}\left\{\sum_{j=1}^{y_{1}}\left[\int_{0}^{t_{j}} C_{1} \exp (-\alpha t) d t+1_{j} \int_{t_{j}}^{t_{j}} C_{2} \exp (-\alpha t) d t\right]\right. \\
& \left.+\sum_{j=1}^{y_{2}} \int_{0}^{s_{1}} C_{2} \exp (-\alpha t) d t\right\} \\
= & \boldsymbol{E}\left\{\sum _ { j = 1 } ^ { y _ { 1 } } \left[\int_{0}^{\infty} C_{1} \exp (-\alpha t) d t-\int_{t_{j}}^{\infty} C_{1} \exp (-\alpha t) d t\right.\right. \\
& \left.+1_{j} \int_{t_{j}}^{\infty} C_{2} \exp (-\alpha t) d t-1_{j} \int_{l_{j}}^{\infty} C_{2} \exp (-\alpha t) d t\right] \\
& \left.+\sum_{j=1}^{y_{2}}\left[\int_{0}^{\infty} C_{2} \exp (-\alpha t) d t-\int_{s_{j}}^{\infty} C_{2} \exp (-\alpha t) d t\right]\right\} .
\end{aligned}
$$

Since $1_{j}$ and $t_{j}$ are independent we get that

$$
\boldsymbol{E}\left\{1_{j} \int_{t_{j}}^{\infty} C_{2} \exp (-\alpha t) d t\right\}=p \boldsymbol{E} \int_{t_{\jmath}}^{\infty} C_{2} \exp (-\alpha t) d t .
$$

Therefore $J^{\pi}\left(y_{1}, y_{2}\right)$ can be written as

$$
\begin{aligned}
& J^{\pi}\left(y_{1}, y_{2}\right) \\
& =K-\frac{1}{\alpha} \boldsymbol{E}\left\{\sum_{j=1}^{y_{1}}\left[\left(C_{1}-p C_{2}\right) \exp \left(-\alpha t_{j}\right)+1_{j} C_{2} \exp \left(-\alpha l_{j}\right)\right]+\sum_{j=1}^{y_{2}} C_{2} \exp \left(-\alpha s_{j}\right)\right\} \\
& \quad=K-\frac{1}{\alpha} R^{\pi}\left(y_{1}, y_{2}\right),
\end{aligned}
$$

where $K$ is some constant. Thus, if a policy $\pi^{*}$ maximizes $R^{\pi}\left(y_{1}, y_{2}\right)$, the same policy minimizes $J^{\pi}\left(y_{1}, y_{2}\right)$.

Let $\tau_{1}, \tau_{2}, \cdots, \tau_{m}$ be the times at which the servers become available. Time $\tau_{i}$, $i=1,2, \cdots, m$, may be the time server $i$ initially becomes available or the time at which server $i$ completes the processing of some job. We define the random variable $r\left(\tau_{1}, \tau_{2}, \cdots, \tau_{m} ; y_{1}, y_{2} ; i_{1}, i_{2}, \cdots, i_{k} ; \pi\right)$ as the reward obtained under policy $\pi$ when the servers become available at times $\tau_{1}, \tau_{2}, \cdots, \tau_{m}$, the total number of jobs to be processed in queue $i, i=1,2$, is $y_{i}$, with $k$ out of $y_{2}$ jobs in queue 2 coming from interconnections from queue 1 at servers $i_{1}, i_{2}, \cdots, i_{k}$ at times $\tau_{i_{1}}, \tau_{i_{2}}, \cdots, \tau_{i_{k}}$. Note that $\left\{i_{1}, i_{2}, \cdots, i_{k}\right\} \subset\{1,2, \ldots, m\}$. We define the random variable $r_{i}\left(\tau_{1}, \tau_{2}\right.$, $\left.\cdots, \tau_{m} ; y_{1}, y_{2} ; i_{1}, i_{2}, \cdots, i_{k} ; \pi\right), i=1,2, \cdots, m$, as the reward obtained on server $i$ when the reward function in queue $i, i=1,2$, is $\dot{g}_{i}(t)$. Finally, $R(\cdot), R_{i}(\cdot)$, $i=1,2, \cdots, m$, denote the expected values of $r(\cdot), r_{i}(\cdot), i=1,2, \cdots, m$, respectively. To define $r_{i}(\cdot)$ and $R_{i}(\cdot)$ without ambiguity we adopt the following conventions: 
(A1) If server $i$ becomes available at the same time as one or more other servers, we assume that it becomes available at a slightly later time.

(A2) If at some time $t$ server $i$ completes the processing of some job (say $j_{l}$ ) in queue 1 and this job joins queue 2 , then server $i$ is guaranteed to process a job at the time $t$ of the interconnection. That is, if $j_{l}$ is the only unassigned job at $t$, server $i$ processes job $j_{l}$.

(A1) is the same as in Weber et al. (1986); (A2) implies that once a server idles because of lack of jobs, it remains idle.

The reward problem we have formulated can be seen as an extension of the work of Weber et al. (1986), where the rewards obtained are the same for all jobs and there are no interconnections between queues. Our basic result is given in the following theorem.

Theorem 1. Suppose that (i) $X_{1} \leqq_{\mathrm{st}} X_{2}$ (where $\leqq_{\mathrm{st}}$ indicates stochastic orderingsee Ross (1983), p. 251), (ii) $X_{1}$ has non-decreasing likelihood ratio, and (iii) $\left(C_{1}-p C_{2}\right) \geqq C_{2}$. Then the non-preemptive scheduling strategy $\mathrm{P} 1$ maximizes the total expected reward within the class of non-preemptive strategies.

The proof of the theorem requires the use of four lemmas.

Lemma 1 . For any scheduling strategy $\pi$

$$
\begin{aligned}
& \frac{d R\left(\tau_{1}, \tau_{2}, \cdots, \tau_{m} ; y_{1}, y_{2} ; i_{1}, i_{2}, \cdots, i_{k} ; \pi\right)}{d \tau_{i}} \\
& =R_{i}\left(\tau_{1}, \tau_{2}, \cdots, \tau_{m} ; y_{1}, y_{2} ; i_{1}, i_{2}, \cdots, i_{k} ; \pi\right), \quad i=1,2, \cdots, m,
\end{aligned}
$$

where $d R(\cdot) / d \tau_{i}$ is the right-hand derivative of $R(\cdot)$ with respect to $\tau_{i}$.

Proof. Let $\left\{\Delta \tau_{n}, n=1,2, \cdots\right\}$ be a sequence of positive real numbers such that $\Delta \tau_{n} \underset{n \rightarrow \infty}{\longrightarrow} 0$, and

$$
\begin{aligned}
Y_{n}= & \left\{r\left(\tau_{1}, \cdots, \tau_{i}+\Delta \tau_{n}, \cdots, \tau_{m} ; y_{1}, y_{2} ; i_{1}, i_{2}, \cdots, i_{k} ; \pi\right)\right. \\
& \left.-r\left(\tau_{1}, \cdots, \tau_{i}, \cdots, \tau_{m} ; y_{1}, y_{2} ; i_{1}, i_{2}, \cdots, i_{k} ; \pi\right)\right\}\left(\Delta \tau_{n}\right)^{-1} .
\end{aligned}
$$

When server $i$ becomes available $\Delta \tau_{n}$ time units later, in the worst case all jobs are delayed by $\Delta \tau_{n}$ time units. Therefore this case provides an upper bound for the numerator of $\left|Y_{n}\right|$. Let $t_{1}, t_{2}, \cdots$ be the service completion times when server $i$ becomes available at time $\tau_{i}$. Then

$$
\begin{aligned}
\left|Y_{n}\right| & \leqq \frac{\sum_{j}\left(K_{j} \exp \left(-\alpha t_{j}\right)-K_{j} \exp \left(-\alpha\left(t_{j}+\Delta \tau_{n}\right)\right)\right)}{\Delta \tau_{n}} \\
& \leqq \alpha \sum_{j} K_{j} \exp \left(-\alpha t_{j}\right)=\alpha r\left(\tau_{1}, \cdots, \tau_{i}, \cdots, \tau_{m} ; y_{1}, y_{2} ; i_{1}, i_{2}, \cdots, i_{k} ; \pi\right),
\end{aligned}
$$


where $K_{j}=C_{1}-p C_{2}$ when the $j$ th completion is at queue 1 and $K_{j}=C_{2}$ when the $j$ th completion is at queue 2 . Taking expectations we get

$$
\boldsymbol{E}\left|Y_{n}\right| \leqq \alpha R\left(\tau_{1}, \cdots, \tau_{i}, \cdots, \tau_{m} ; y_{1}, y_{2} ; i_{1}, i_{2}, \cdots, i_{k} ; \pi\right)<\infty .
$$

For any sample path of service times and interconnections, and for $n$ sufficiently large $\left(\Delta \tau_{n}\right.$ sufficiently small), all jobs are processed by the same servers when server $i$ becomes available at times $\tau_{i}$ and $\tau_{i}+\Delta \tau_{n}$. Therefore, with $t_{1}, t_{2}, \cdots$ denoting now the service completion times at server $i$ when it becomes available at time $\tau_{i}$, we have for $n$ sufficiently large

$$
Y_{n}=\frac{\sum_{j}\left(K_{j} \exp \left(-\alpha\left(t_{j}+\Delta \tau_{n}\right)\right)-K_{j} \exp \left(-\alpha t_{j}\right)\right)}{\Delta \tau_{n}},
$$

where $K_{j}=C_{1}-p C_{2}$ or $C_{2}$ depending on the queue the $j$ th completion takes place. Taking limits we get

$$
Y_{n} \underset{n \rightarrow \infty}{\longrightarrow} \sum_{j}-\alpha K_{j} \exp \left(-\alpha t_{j}\right)=r_{i}\left(\tau_{1}, \cdots, \tau_{i}, \cdots, \tau_{m} ; y_{1}, y_{2} ; i_{1}, i_{2}, \cdots, i_{k} ; \pi\right) .
$$

From (2.1), (2.2) and the dominated convergence theorem we obtain

$$
\begin{aligned}
\boldsymbol{E} Y_{n} & \underset{n \rightarrow \infty}{\longrightarrow} \boldsymbol{E}\left[r_{i}\left(\tau_{1}, \cdots, \tau_{i}, \cdots, \tau_{m} ; y_{1}, y_{2} ; i_{1}, i_{2}, \cdots, i_{k} ; \pi\right)\right] \\
& =R_{i}\left(\tau_{1}, \cdots, \tau_{i}, \cdots, \tau_{m} ; y_{1}, y_{2} ; i_{1}, i_{2}, \cdots, i_{k} ; \pi\right) .
\end{aligned}
$$

Since this is true for any positive real sequence $\Delta \tau_{n}$ for which $\Delta \tau_{n} \underset{n \rightarrow \infty}{\longrightarrow} 0$, we conclude that

$$
\begin{aligned}
\lim _{\Delta \tau \downarrow 0} \boldsymbol{E}\{r & \left(\tau_{1}, \cdots, \tau_{i}+\Delta \tau, \cdots, \tau_{m} ; y_{1}, y_{2} ; i_{1}, i_{2}, \cdots, i_{k} ; \pi\right) \\
& \left.\quad r\left(\tau_{1}, \cdots, \tau_{i}, \cdots, \tau_{m} ; y_{1}, y_{2} ; i_{1}, i_{2}, \cdots, i_{k} ; \pi\right)\right\}(\Delta \tau)^{-1}=\frac{d R(\cdot)}{d \tau_{i}}=R_{i}(\cdot) .
\end{aligned}
$$

The result of Lemma 1 is very important for our development. In essence, Lemma 1 allows us to convert an $m$-server problem into $m$ one-server problems.

Lemma 2. Suppose strategy $\mathrm{P} 1$ is employed. Then

(a) $R_{1}\left(\tau_{1}, \tau_{2}, \cdots, \tau_{m} ; y_{1}, y_{2} ; 1, i_{1}, i_{2}, \cdots, i_{k} ; \mathrm{P} 1\right) \leqq R_{1}\left(\tau_{1}, \tau_{2}, \cdots, \tau_{m} ; y_{1}, y_{2} ; i_{1}\right.$, $\left.i_{2}, \cdots, i_{k} ; \mathrm{P} 1\right)$

(b) $R_{1}\left(\tau_{1}, \tau_{2}, \cdots, \tau_{m} ; y_{1}, y_{2} ; 2, i_{1}, i_{2}, \cdots, i_{k} ; \mathrm{P} 1\right) \geqq R_{1}\left(\tau_{1}, \tau_{2}, \cdots, \tau_{m} ; y_{1}, y_{2} ; i_{1}\right.$, $\left.i_{2}, \cdots, i_{k} ; \mathrm{P} 1\right)$. 


\section{Proof.}

(a) Without loss of generality, let $\tau_{2}=\min _{i \neq 1} \tau_{i}$. If $\tau_{1}<\tau_{2}$, the inequality is satisfied as an equality, so we only have to consider the case $\tau_{1} \geqq \tau_{2}$. The proof is by induction on $y_{1}$. We first prove the result for $y_{1}=0$ and any $y_{2}$ by induction on $y_{2}$. For $y_{1}=0, y_{2}=1, R_{1}\left(\tau_{1}, \tau_{2}, \cdots, \tau_{m} ; 0,1 ;-; \mathrm{P} 1\right)$ is zero because the job in queue 2 is served by server 2 , while $R_{1}\left(\tau_{1}, \tau_{2}, \cdots, \tau_{m} ; 0,1 ; 1 ; \mathrm{P} 1\right)$ is negative. We assume that the result is true for $y_{1}=0, y_{2}=l-1$, and prove it for $y_{1}=0, y_{2}=l$.

Consider $R_{1}\left(\tau_{1}, \tau_{2}, \cdots, \tau_{m} ; 0, l ; 1, i_{1}, i_{2}, \cdots, i_{k} ; \mathrm{P} 1\right)$. Notice that $\tau_{1}$ is an interconnection time. If server 1 is the first to process a job, then the rest of the jobs come from interconnections after $\tau_{1}$. In that case $R_{1}\left(\tau_{1}, \tau_{2}, \cdots, \tau_{m}\right.$; $\left.0, l ; 1, i_{1}, i_{2}, \cdots, i_{k} ; \mathrm{P} 1\right)<0$ and $R_{1}\left(\tau_{1}, \tau_{2}, \cdots, \tau_{m} ; 0, l ; i_{1}, i_{2}, \cdots, i_{k} ; \mathrm{P} 1\right)=0$ because of (A2). If server $i, i \neq 1$, is the first to process a job, then

$$
\begin{aligned}
& R_{1}\left(\tau_{1}, \tau_{2}, \cdots, \tau_{m} ; 0, l ; 1, i_{1}, i_{2}, \cdots, i_{k} ; \mathrm{P} 1\right) \\
& \quad=\mathbf{E}\left\{R_{1}\left(\tau_{1}, \cdots, \tau_{i}+X_{2}, \cdots, \tau_{m} ; 0, l-1 ; 1, i_{1}, \cdots, i_{k} ; \mathrm{P} 1\right)\right\} \\
& \quad \leqq E\left\{R_{1}\left(\tau_{1}, \cdots, \tau_{i}+X_{2}, \cdots, \tau_{m} ; 0, l-1 ; i_{1}, \cdots, i_{k} ; \mathrm{P} 1\right)\right\} \\
& \quad=R_{1}\left(\tau_{1}, \tau_{2}, \cdots, \tau_{m} ; 0, l ; i_{1}, i_{2}, \cdots, i_{k} ; \mathrm{P} 1\right),
\end{aligned}
$$

where the inequality follows from the induction hypothesis.

We now assume that the result is true for $y_{1}=l-1$ and any $y_{2}$, and prove it for $y_{1}=l$ and any $y_{2}$. Since server 2 processes a job in queue 1 , we have

$$
\begin{aligned}
& R_{1}\left(\tau_{1}, \tau_{2}, \cdots, \tau_{m} ; l, y_{2} ; 1, i_{1}, i_{2}, \cdots, i_{k} ; \mathrm{P} 1\right) \\
&= \boldsymbol{E}\left\{p R_{1}\left(\tau_{1}, \tau_{2}+X_{1}, \cdots, \tau_{m} ; l-1, y_{2}+1 ; 1,2, i_{1}, i_{2}, \cdots, i_{k} ; \mathrm{P} 1\right)\right. \\
&\left.+(1-p) R_{1}\left(\tau_{1}, \tau_{2}+X_{1}, \cdots, \tau_{m} ; l-1, y_{2} ; 1, i_{1}, i_{2}, \cdots, i_{k} ; \mathrm{P} 1\right)\right\} \\
& \leqq \boldsymbol{E}\left\{p R_{1}\left(\tau_{1}, \tau_{2}+X_{1}, \cdots, \tau_{m} ; l-1, y_{2}+1 ; 2, i_{1}, i_{2}, \cdots, i_{k} ; \mathrm{P} 1\right)\right. \\
&\left.+(1-p) R_{1}\left(\tau_{1}, \tau_{2}+X_{1}, \cdots, \tau_{m} ; l-1, y_{2} ; i_{1}, i_{2}, \cdots, i_{k} ; \mathrm{P} 1\right)\right\} \\
&= R_{1}\left(\tau_{1}, \tau_{2}, \cdots, \tau_{m} ; l, y_{2} ; i_{1}, i_{2}, \cdots, i_{k} ; \mathrm{P} 1\right),
\end{aligned}
$$

where the inequality follows from the induction hypothesis.

(b) Notice that if $\tau_{2}=\min _{i} \tau_{i}$, the inequality is satisfied as an equality. We assume that $\tau_{2} \neq \min _{i} \tau_{i}$. We use the same induction scheme as in part (a). For $y_{1}=0, y_{2}=1$, $R_{1}\left(\tau_{1}, \tau_{2}, \cdots, \tau_{m} ; 0,1 ; 2 ; \mathrm{P} 1\right)$ is zero because the job in queue 2 is processed by server 2 , while $R_{1}\left(\tau_{1}, \tau_{2}, \cdots, \tau_{m} ; 0,1 ;-; \mathrm{P} 1\right)$ is non-positive. We assume that the result is true for $y_{1}=0, y_{2}=l-1$ and prove it for $y_{1}=0, y_{2}=l$.

Consider $R_{1}\left(\tau_{1}, \tau_{2}, \cdots, \tau_{m} ; 0, l ; 2, i_{1}, i_{2}, \cdots, i_{k} ; \mathrm{P} 1\right)$. For $\tau_{1} \geqq \tau_{2}$, if server 2 is the first to process a job, the rest of the jobs come from interconnections after $\tau_{2}$. If there is no interconnection at time $\tau_{1}$,

$$
\begin{aligned}
R_{1}\left(\tau_{1}, \tau_{2}, \cdots, \tau_{m} ; 0, l ; 2, i_{1}, i_{2}, \cdots, i_{k} ; \mathrm{P} 1\right) \\
\quad=R_{1}\left(\tau_{1}, \tau_{2}, \cdots, \tau_{m} ; 0, l ; i_{1}, i_{2}, \cdots, i_{k} ; \mathrm{P} 1\right)=0 .
\end{aligned}
$$


If there is an interconnection at time $\tau_{1}$, this job is processed by server 1 and

$$
\begin{aligned}
& R_{1}\left(\tau_{1}, \tau_{2}, \cdots, \tau_{m} ; 0, l ; 2, i_{1}, i_{2}, \cdots, i_{k} ; \mathrm{P} 1\right) \\
& \quad=\boldsymbol{E}\left\{\dot{g}_{2}\left(\tau_{1}+X_{2}\right)+R_{1}\left(\tau_{1}+X_{2}, \tau_{2}, \cdots, \tau_{m} ; 0, l-1 ; 2, i_{1}, i_{2}, \cdots, i_{k} ; \mathrm{P} 1\right)\right\} \\
& \quad \geqq \boldsymbol{E}\left\{\dot{g}_{2}\left(\tau_{1}+X_{2}\right)+R_{1}\left(\tau_{1}+X_{2}, \tau_{2}, \cdots, \tau_{m} ; 0, l-1 ; i_{1}, i_{2}, \cdots, i_{k} ; \mathrm{P} 1\right)\right\} \\
& \quad=R_{1}\left(\tau_{1}, \tau_{2}, \cdots, \tau_{m} ; 0, l ; i_{1}, i_{2}, \cdots, i_{k} ; \mathrm{P} 1\right),
\end{aligned}
$$

where the inequality follows from the induction hypothesis. If server $i, i \neq 2$, is the first to process a job, $\tau_{i} \leqq \tau_{2}$ has to be true, implying $i \neq 1$. Then

$$
\begin{aligned}
& R_{1}\left(\tau_{1}, \tau_{2}, \cdots, \tau_{m} ; 0, l ; 2, i_{1}, i_{2}, \cdots, i_{k} ; \mathrm{P} 1\right) \\
& \quad=\boldsymbol{E}\left\{R_{1}\left(\tau_{1}, \tau_{2}, \cdots, \tau_{i}+X_{2}, \cdots, \tau_{m} ; 0, l-1 ; 2, i_{1}, i_{2}, \cdots, i_{k} ; \mathrm{P} 1\right)\right\} \\
& \quad \geqq \boldsymbol{E}\left\{R_{1}\left(\tau_{1}, \tau_{2}, \cdots, \tau_{i}+X_{2}, \cdots, \tau_{m} ; 0, l-1 ; i_{1}, i_{2}, \cdots, i_{k} ; \mathrm{P} 1\right)\right\} \\
& \quad=R_{1}\left(\tau_{1}, \tau_{2}, \cdots, \tau_{m} ; 0, l ; i_{1}, i_{2}, \cdots, i_{k} ; \mathrm{P} 1\right),
\end{aligned}
$$

where the inequality follows from the induction hypothesis.

For $\tau_{1}<\tau_{2}$, if server 2 is the first to process a job, we get $R_{1}\left(\tau_{1}, \tau_{2}, \cdots, \tau_{m} ; 0, l ; 2, i_{1}, i_{2}, \cdots, i_{k} ; \mathrm{P} 1\right)=0$, since all other jobs come from interconnections after $\tau_{2}$. If server $i, i \neq 2$, is the first to process a job, the result follows from the induction hypothesis in the same way as in the case $\tau_{1} \geqq \tau_{2}$.

We now assume that the result is true for $y_{1}=l-1$ and any $y_{2}$, and prove it for $y_{1}=l$ and any $y_{2}$. We prove the result using the induction hypothesis in the same way as in part (a).

The intuition behind Lemma 2 is that, since the reward function $\dot{g}_{i}(t), i=1,2$, is negative, the reward obtained on a given server (server 1 without loss of generality) is not increased when there is at least one job for that server to process (job coming from an interconnection at time $\tau_{1}$ in part (a)), and is not decreased when there is at least one job for some other server to process (job coming from an interconnection at time $\tau_{2}$ in part (b)).

Lemma 3. Suppose strategy $\mathrm{P} 1$ is employed. Then $R_{i}\left(\tau_{1}, \cdots, \tau_{i}, \cdots, \tau_{m}\right.$; $\left.y_{1}, y_{2} ; i_{1}, i_{2}, \cdots, i_{k} ; \mathrm{P} 1\right)$ is (a) non-decreasing in $\tau_{i}$ and (b) non-increasing in $\tau_{j}, j \neq i$.

Proof. Without loss of generality we prove the lemma for $i=1$. The result (parts (a) and (b)) is trivially true for $y_{1}=0, y_{2}=0$. We assume that it is true for $y_{1}=0$, $y_{2}=l-1$ and prove it for $y_{1}=0, y_{2}=l$. We give the proof for part (a) only, since part (b) can be proved similarly. We assume that $\tau_{2}=\min _{i \neq 1} \tau_{i}$. If there is an interconnection at time $\tau_{1}\left(\tau_{1}=\tau_{i}\right.$ for some $\left.j, 1 \leqq j \leqq k\right)$, we have

$$
\begin{aligned}
& R_{1}\left(\tau_{1}, \tau_{2}, \cdots, \tau_{m} ; 0, l ; i_{1}, i_{2}, \cdots, i_{k} ; \mathrm{P} 1\right) \\
& =\boldsymbol{E}\left\{\dot{g}_{2}\left(\tau_{1}+X_{2}\right)+R_{1}\left(\tau_{1}+X_{2}, \tau_{2}, \cdots, \tau_{m} ; 0, l-1 ; i_{1}, i_{2}, \cdots, i_{j-1}, i_{j+1}, \cdots, i_{k} ; \mathrm{P} 1\right)\right\},
\end{aligned}
$$


and the result follows from $\dot{g}_{2}(t)$ being non-decreasing and the induction hypothesis. If there is no interconnection at time $\tau_{1}$ and all $l$ jobs in queue 2 come from interconnections, then $R_{1}\left(\tau_{1}, \tau_{2}, \cdots, \tau_{m} ; 0, l ; i_{1}, i_{2}, \cdots, i_{k} ; \mathrm{P} 1\right)=0$. Consider the case when there is no interconnection at time $\tau_{1}$ and not all $l$ jobs in queue 2 come from interconnections. Then for $\tau_{1}<\tau_{2}$,

$$
\begin{aligned}
R_{1}\left(\tau_{1}, \tau_{2}, \cdots, \tau_{m} ; 0, l ; i_{1}, i_{2}, \cdots, i_{k} ; \mathrm{P} 1\right) \\
=\boldsymbol{E}\left\{\dot{g}_{2}\left(\tau_{1}+X_{2}\right)+R_{1}\left(\tau_{1}+X_{2}, \tau_{2}, \cdots, \tau_{m} ; 0, l-1 ; i_{1}, i_{2}, \cdots, i_{k} ; \mathrm{P} 1\right)\right\},
\end{aligned}
$$

and for $\tau_{1}>\tau_{2}$

$$
\begin{aligned}
& R_{1}\left(\tau_{1}, \tau_{2}, \cdots, \tau_{m} ; 0, l ; i_{1}, i_{2}, \cdots, i_{k} ; \mathrm{P} 1\right) \\
& \qquad \boldsymbol{E}\left\{R_{1}\left(\tau_{1}, \tau_{2}+X_{2}, \cdots, \tau_{m} ; 0, l-1 ; i_{1}, i_{2}, \cdots, i_{k} ; \mathrm{P} 1\right)\right\} .
\end{aligned}
$$

The result follows from $\dot{g}_{2}(t)$ being non-decreasing and the induction hypothesis. It remains to be seen how $R_{1}(\cdot)$ changes when $\tau_{1}$ passes through the value $\tau_{2}$. This change is given by $R_{1}\left(\tau_{2}^{+}, \tau_{2}, \cdots, \tau_{m} ; 0, l ; i_{1}, i_{2}, \cdots, i_{k} ; \mathrm{P} 1\right)-R_{1}\left(\tau_{2}^{-}, \tau_{2}, \cdots, \tau_{m}\right.$; $\left.0, l ; i_{1}, i_{2}, \cdots, i_{k} ; \mathrm{P} 1\right)$. When there is a job to be processed by server 1 when $\tau_{1}=\tau_{2}^{+}$, the difference is zero. When there is no job to be processed by server 1 when $\tau_{1}=\tau_{2}^{+}$, the difference is non-negative because the first term is zero and the second is non-positive.

We now assume that Lemma 3 (parts (a) and (b)) is true for $y_{1}=l-1$ and any $y_{2}$, and prove it for $y_{1}=l$ and any $y_{2}$. Again we give the proof for part (a) only, assuming $\tau_{2}=\min _{i \neq 1} \tau_{i}$. For $\tau_{1}<\tau_{2}$,

$$
\begin{aligned}
R_{1}\left(\tau_{1}, \tau_{2}, \cdots, \tau_{m} ; l, y_{2} ; i_{1}, i_{2}, \cdots, i_{k} ; \mathrm{P} 1\right) \\
=\boldsymbol{E}\left\{\dot{g}_{1}\left(\tau_{1}+X_{1}\right)+p R_{1}\left(\tau_{1}+X_{1}, \tau_{2}, \cdots, \tau_{m} ; l-1, y_{2}+1 ; 1, i_{1}, i_{2}, \cdots, i_{k} ; \mathrm{P} 1\right)\right. \\
\left.+(1-p) R_{1}\left(\tau_{1}+X_{1}, \tau_{2}, \cdots, \tau_{m} ; l-1, y_{2} ; i_{1}, i_{2}, \cdots, i_{k} ; \mathrm{P} 1\right)\right\},
\end{aligned}
$$

and for $\tau_{1}>\tau_{2}$,

$$
\begin{aligned}
& R_{1}\left(\tau_{1}, \tau_{2}, \cdots, \tau_{m} ; l, y_{2} ; i_{1}, i_{2}, \cdots, i_{k} ; \mathrm{P} 1\right) \\
& =\boldsymbol{E}\left\{p R_{1}\left(\tau_{1}, \tau_{2}+X_{1}, \cdots, \tau_{m} ; l-1, y_{2}+1 ; 2, i_{1}, i_{2}, \cdots, i_{k} ; \mathrm{P} 1\right)\right. \\
& \left.\quad+(1-p) R_{1}\left(\tau_{1}, \tau_{2}+X_{1}, \cdots, \tau_{m} ; l-1, y_{2} ; i_{1}, i_{2}, \cdots, i_{k} ; \mathrm{P} 1\right)\right\} .
\end{aligned}
$$

The result follows from $\dot{g}_{1}(t)$ being non-decreasing and the induction hypothesis. It remains to show that

$$
R_{1}\left(\tau_{2}^{+}, \tau_{2}, \cdots, \tau_{m} ; l, y_{2} ; i_{1}, i_{2}, \cdots, i_{k} ; \mathrm{P} 1\right)
$$

$$
\geqq R_{1}\left(\tau_{2}^{-}, \tau_{2}, \cdots, \tau_{m} ; l, y_{2} ; i_{1}, i_{2}, \cdots, i_{k} ; \mathrm{P} 1\right) .
$$

If there is a job in queue 1 to be processed by server 1 when $\tau_{1}=\tau_{2}^{+}$, the two rewards are the same. If there is no job to be processed by server 1 when $\tau_{1}=\tau_{2}^{+}$, 
$R_{1}\left(\tau_{2}^{+}, \tau_{2}, \cdots, \tau_{m} ; l, y_{2} ; i_{1}, i_{2}, \cdots, i_{k} ; \mathrm{P} 1\right)$ is zero and the inequality is satisfied. If server 1 processes a job in queue 2 when $\tau_{1}=\tau_{2}^{+}$, we have

$$
\begin{aligned}
& R_{1}\left(\tau_{2}^{+}, \tau_{2}, \cdots, \tau_{m} ; l, y_{2} ; i_{1}, i_{2}, \cdots, i_{k} ; \mathrm{P} 1\right) \\
& =\boldsymbol{E}\left\{\dot{g}_{2}\left(\tau_{2}+X_{2}\right)+p R_{1}\left(\tau_{2}+X_{2}, \tau_{2}+X_{1}, \cdots, \tau_{m} ; l-1, y_{2} ; 2, i_{1}, i_{2}, \cdots, i_{k} ; \mathrm{P} 1\right)\right. \\
& \left.\quad+(1-p) R_{1}\left(\tau_{2}+X_{2}, \tau_{2}+X_{1}, \cdots, \tau_{m} ; l-1, y_{2}-1 ; i_{1}, i_{2}, \cdots, i_{k} ; \mathrm{P} 1\right)\right\} \\
& R_{1}\left(\tau_{2}^{-}, \tau_{2}, \cdots, \tau_{m} ; l, y_{2} ; i_{1}, i_{2}, \cdots, i_{k} ; \mathrm{P} 1\right) \\
& \quad=\boldsymbol{E}\left\{\dot{g}_{1}\left(\tau_{2}+X_{1}\right)+p R_{1}\left(\tau_{2}+X_{1}, \tau_{2}+X_{2}, \cdots, \tau_{m} ; l-1, y_{2} ; i_{1}, i_{2}, \cdots, i_{k} ; \mathrm{P} 1\right)\right. \\
& \left.\quad+(1-p) R_{1}\left(\tau_{2}+X_{1}, \tau_{2}+X_{2}, \cdots, \tau_{m} ; l-1, y_{2}-1 ; i_{1}, i_{2}, \cdots, i_{k} ; \mathrm{P} 1\right)\right\}
\end{aligned}
$$

From conditions (i) and (iii) in the theorem we get

$$
\boldsymbol{E}\left\{\dot{g}_{2}\left(\tau_{2}+X_{2}\right)\right\} \geqq \boldsymbol{E}\left\{\dot{g}_{1}\left(\tau_{2}+X_{1}\right)\right\}
$$

From condition (i) in the theorem $\left(X_{1} \leqq_{\mathrm{st}} X_{2}\right)$ and the induction hypothesis for parts (a) and (b) we get

$$
\begin{aligned}
& \boldsymbol{E}\left\{R_{1}\left(\tau_{2}+X_{2}, \tau_{2}+X_{1}, \cdots, \tau_{m} ; l-1, y_{2}-1 ; i_{1}, i_{2}, \cdots, i_{k} ; \mathrm{P} 1\right)\right\} \\
& \quad \geqq \boldsymbol{E}\left\{R_{1}\left(\tau_{2}+X_{1}, \tau_{2}+X_{2}, \cdots, \tau_{m} ; l-1, y_{2}-1 ; i_{1}, i_{2}, \cdots, i_{k} ; \mathrm{P} 1\right)\right\} .
\end{aligned}
$$

Finally we have

$$
\begin{aligned}
& \boldsymbol{E}\left\{R_{1}\left(\tau_{2}+X_{2}, \tau_{2}+X_{1}, \cdots, \tau_{m} ; l-1, y_{2} ; 2, i_{1}, i_{2}, \cdots, i_{k} ; \mathrm{P} 1\right)\right\} \\
& \quad \geqq \boldsymbol{E}\left\{R_{1}\left(\tau_{2}+X_{2}, \tau_{2}+X_{1}, \cdots, \tau_{m} ; l-1, y_{2} ; i_{1}, i_{2}, \cdots, i_{k} ; \mathrm{P} 1\right)\right\} \\
& \quad \geqq \boldsymbol{E}\left\{R_{1}\left(\tau_{2}+X_{1}, \tau_{2}+X_{2}, \cdots, \tau_{m} ; l-1, y_{2} ; i_{1}, i_{2}, \cdots, i_{k} ; \mathrm{P} 1\right)\right\} \\
& \quad \geqq \boldsymbol{E}\left\{R_{1}\left(\tau_{2}+X_{1}, \tau_{2}+X_{2}, \cdots, \tau_{m} ; l-1, y_{2} ; 1, i_{1}, i_{2}, \cdots, i_{k} ; \mathrm{P} 1\right)\right\},
\end{aligned}
$$

where the first and last inequalities follow from Lemmas $2 \mathrm{~b}$ and $2 \mathrm{a}$ respectively, and the middle one is (2.7) with $y_{2}-1$ replaced by $y_{2}$. Equation (2.3) follows from Equations (2.4)-(2.8).

The intuition behind Lemma 3 is the following. When a server $i$ becomes 
available at a later time, it either processes fewer jobs or processes the same jobs later or processes jobs in queue 2 instead of queue 1 . Since the reward function $\dot{g}_{i}(t)$, $i=1,2$, is negative and non-decreasing, and the expected reward obtained in queue 2 is greater than the expected reward obtained in queue 1 (see conditions (i) and (iii) in the theorem), the expected reward obtained on server $i$ does not decrease. Similarly, when server $j, j \neq i$, becomes available at a later time, server $i$ either processes more jobs or processes jobs in queue 1 instead of queue 2 . Therefore, the expected reward obtained on server $i$ does not increase.

The final lemma states that when the reward function is $\dot{g}_{i}(t), i=1,2$, the expected reward obtained on a server that does not start first is greater when employing strategy P1 than when employing a strategy that is identical to P1 except that it assigns a job in queue 1 (if any) to that server.

Lemma 4. Let $\tau_{1} \neq \min _{i} \tau_{i}$. If there is no interconnection at time $\tau_{1}$, we have:

(a) If not all jobs in queue 2 come from interconnections

$$
\begin{aligned}
& R_{1}\left(\tau_{1}, \tau_{2}, \cdots, \tau_{m} ; 0, y_{2} ; i_{1}, i_{2}, \cdots, i_{k} ; \mathrm{P} 1\right) \\
& \quad \geqq E \\
& \quad
\end{aligned}
$$

$$
\begin{aligned}
& R_{1}\left(\tau_{1}, \tau_{2}, \cdots, \tau_{m} ; y_{1}, y_{2} ; i_{1}, i_{2}, \cdots, i_{k} ; \mathrm{P} 1\right) \geqq \boldsymbol{E}\left\{\dot{g}_{1}\left(\tau_{1}+X_{1}\right)\right. \\
& \quad+p R_{1}\left(\tau_{1}+X_{1}, \tau_{2}, \cdots, \tau_{m} ; y_{1}-1, y_{2}+1 ; 1, i_{1}, i_{2}, \cdots, i_{k} ; \mathrm{P} 1\right) \\
& \left.\quad+(1-p) R_{1}\left(\tau_{1}+X_{1}, \tau_{2}, \cdots, \tau_{m} ; y_{1}-1, y_{2} ; i_{1}, i_{2}, \cdots, i_{k} ; \mathrm{P} 1\right)\right\}, \quad y_{1} \geqq 1 .
\end{aligned}
$$

Proof. Without loss of generality we assume that $\tau_{2}=\min _{i} \tau_{i}$.

(a) We prove the result by induction on $y_{2}$. The result is true for $y_{2}=1$ since the left-hand side of (2.9) is zero. We assume that it is true for $y_{2}=l-1$ and prove it for $y_{2}=l$. Because not all jobs in queue 2 come from interconnections we have

$$
\begin{aligned}
R_{1}\left(\tau_{1}, \tau_{2}, \cdots, \tau_{m} ; 0, l ; i_{1}, i_{2}, \cdots, i_{k} ; \mathrm{P} 1\right) \\
\qquad \boldsymbol{E}\left\{R_{1}\left(\tau_{1}, \tau_{2}+X_{2}, \cdots, \tau_{m} ; 0, l-1 ; i_{1}, i_{2}, \cdots, i_{k} ; \mathrm{P} 1\right)\right\} .
\end{aligned}
$$

If all $l-1$ jobs in queue 2 come from interconnections, the last term is zero. If not, we can use the induction hypothesis to get

$$
\begin{aligned}
& R_{1}\left(\tau_{1}, \tau_{2}, \cdots, \tau_{m} ; 0, l ; i_{1}, i_{2}, \cdots, i_{k} ; \mathrm{P} 1\right) \\
& \quad \geqq \boldsymbol{E}\left\{\dot{g}_{2}\left(\tau_{1}+\tilde{X}_{2}\right)+R_{1}\left(\tau_{1}+\tilde{X}_{2}, \tau_{2}+X_{2}, \cdots, \tau_{m} ; 0, l-2 ; i_{1}, i_{2}, \cdots, i_{k} ; \mathrm{P} 1\right)\right\} \\
& \quad=\boldsymbol{E}\left\{\dot{g}_{2}\left(\tau_{1}+\tilde{X}_{2}\right)+R_{1}\left(\tau_{1}+\tilde{X}_{2}, \tau_{2}, \cdots, \tau_{m} ; 0, l-1 ; i_{1}, i_{2}, \cdots, i_{k} ; \mathrm{P} 1\right)\right\},
\end{aligned}
$$

where $\tilde{X}_{2}$ is a random variable independent of and identically distributed to $X_{2}$. 
(b) The result is true for $y_{1}=1, y_{2}=0$ since the left-hand side of (2.10) is zero. For $y_{2} \neq 0$ we have

$$
\begin{aligned}
& R_{1}\left(\tau_{1}, \tau_{2}, \cdots, \tau_{m} ; 1, y_{2} ; i_{1}, i_{2}, \cdots, i_{k} ; \mathrm{P} 1\right) \\
&= \boldsymbol{E}\left\{p R_{1}\left(\tau_{1}, \tau_{2}+X_{1}, \cdots, \tau_{m} ; 0, y_{2}+1 ; 2, i_{1}, i_{2}, \cdots, i_{k} ; \mathrm{P} 1\right)\right. \\
&\left.+(1-p) R_{1}\left(\tau_{1}, \tau_{2}+X_{1}, \cdots, \tau_{m} ; 0, y_{2} ; i_{1}, i_{2}, \cdots, i_{k} ; \mathrm{P} 1\right)\right\} .
\end{aligned}
$$

If all $y_{2}$ jobs in queue 2 come from interconnections, the last term is zero. If not, we can use part (a) of the lemma to get

$$
\begin{aligned}
& R_{1}\left(\tau_{1}, \tau_{2}, \cdots, \tau_{m} ; 1, y_{2} ; i_{1}, i_{2}, \cdots, i_{k} ; \mathrm{P} 1\right) \\
& \geqq \boldsymbol{E}\left\{\dot{g}_{2}\left(\tau_{1}+X_{2}\right)+p R_{1}\left(\tau_{1}+X_{2}, \tau_{2}+X_{1}, \cdots, \tau_{m} ; 0, y_{2} ; 2, i_{1}, i_{2}, \cdots, i_{k} ; \mathrm{P} 1\right)\right. \\
&\left.+(1-p) R_{1}\left(\tau_{1}+X_{2}, \tau_{2}+X_{1}, \cdots, \tau_{m} ; 0, y_{2}-1 ; i_{1}, i_{2}, \cdots, i_{k} ; \mathrm{P} 1\right)\right\} \\
& \geqq \boldsymbol{E}\left\{\dot{g}_{1}\left(\tau_{1}+X_{1}\right)+p R_{1}\left(\tau_{1}+X_{1}, \tau_{2}+X_{2}, \cdots, \tau_{m} ; 0, y_{2} ; 2, i_{1}, i_{2}, \cdots, i_{k} ; \mathrm{P} 1\right)\right. \\
&\left.+(1-p) R_{1}\left(\tau_{1}+X_{1}, \tau_{2}+X_{2}, \cdots, \tau_{m} ; 0, y_{2}-1 ; i_{1}, i_{2}, \cdots, i_{k} ; \mathrm{P} 1\right)\right\} \\
& \geqq \boldsymbol{E}\left\{\dot{g}_{1}\left(\tau_{1}+X_{1}\right)+p R_{1}\left(\tau_{1}+X_{1}, \tau_{2}+X_{2}, \cdots, \tau_{m} ; 0, y_{2} ; 1, i_{1}, i_{2}, \cdots, i_{k} ; \mathrm{P} 1\right)\right. \\
&\left.+(1-p) R_{1}\left(\tau_{1}+X_{1}, \tau_{2}+X_{2}, \cdots, \tau_{m} ; 0, y_{2}-1 ; i_{1}, i_{2}, \cdots, i_{k} ; \mathrm{P} 1\right)\right\} \\
&= \boldsymbol{E}\left\{\dot{g}_{1}\left(\tau_{1}+X_{1}\right)+p R_{1}\left(\tau_{1}+X_{1}, \tau_{2}, \cdots, \tau_{m} ; 0, y_{2}+1 ; 1, i_{1}, i_{2}, \cdots, i_{k} ; \mathrm{P} 1\right)\right. \\
&\left.+(1-p) R_{1}\left(\tau_{1}+X_{1}, \tau_{2}, \cdots, \tau_{m} ; 0, y_{2} ; i_{1}, i_{2}, \cdots, i_{k} ; \mathrm{P} 1\right)\right\},
\end{aligned}
$$

where the first inequality follows from Lemma 3 and the second inequality follows from applying first Lemma $2 \mathrm{~b}$ and then Lemma $2 \mathrm{a}$.

We now assume that the result is true for $y_{1}=l-1$ and any $y_{2}$ and prove it for $y_{1}=l$ and any $y_{2}$. We have

$$
\begin{aligned}
& R_{1}\left(\tau_{1}, \tau_{2}, \cdots, \tau_{m} ; l, y_{2} ; i_{1}, i_{2}, \cdots, i_{k} ; \mathrm{P} 1\right) \\
&= \boldsymbol{E}\left\{p R_{1}\left(\tau_{1}, \tau_{2}+X_{1}, \cdots, \tau_{m} ; l-1, y_{2}+1 ; 2, i_{1}, i_{2}, \cdots, i_{k} ; \mathrm{P} 1\right)\right. \\
&\left.+(1-p) R_{1}\left(\tau_{1}, \tau_{2}+X_{1}, \cdots, \tau_{m} ; l-1, y_{2} ; i_{1}, i_{2}, \cdots, i_{k} ; \mathrm{P} 1\right)\right\} \\
& \geqq \boldsymbol{E}\left\{\dot{g}_{1}\left(\tau_{1}+\tilde{X}_{1}\right)\right. \\
&+p\left[p R_{1}\left(\tau_{1}+\tilde{X}_{1}, \tau_{2}+X_{1}, \cdots, \tau_{m} ; l-2, y_{2}+2 ; 1,2 ; i_{1}, i_{2}, \cdots, i_{k} ; \mathrm{P} 1\right)\right. \\
&\left.+(1-p) R_{1}\left(\tau_{1}+\tilde{X}_{1}, \tau_{2}+X_{1}, \cdots, \tau_{m} ; l-2, y_{2}+1 ; 2, i_{1}, i_{2}, \cdots, i_{k} ; \mathrm{P} 1\right)\right] \\
&+(1-p)\left[p R_{1}\left(\tau_{1}+\tilde{X}_{1}, \tau_{2}+X_{1}, \cdots, \tau_{m} ; l-2, y_{2}+1 ; 1, i_{1}, i_{2}, \cdots, i_{k} ; \mathrm{P} 1\right)\right. \\
&\left.\left.+(1-p) R_{1}\left(\tau_{1}+\tilde{X}_{1}, \tau_{2}+X_{1}, \cdots, \tau_{m} ; l-2, y_{2} ; i_{1}, i_{2}, \cdots, i_{k} ; \mathrm{P} 1\right)\right]\right\},
\end{aligned}
$$


where the inequality follows from the induction hypothesis. Rearranging terms, the last expression can be written as

$$
\begin{aligned}
\boldsymbol{E}\left\{\dot{g}_{1}\left(\tau_{1}+\tilde{X}_{1}\right)\right. & \\
& +p\left[p R_{1}\left(\tau_{1}+\tilde{X}_{1}, \tau_{2}+X_{1}, \cdots, \tau_{m} ; l-2, y_{2}+2 ; 1,2, i_{1}, i_{2}, \cdots, i_{k} ; \mathrm{P} 1\right)\right. \\
& \left.+(1-p) R_{1}\left(\tau_{1}+\tilde{X}_{1}, \tau_{2}+X_{1}, \cdots, \tau_{m} ; l-2, y_{2}+1 ; 1, i_{1}, i_{2}, \cdots, i_{k} ; \mathrm{P} 1\right)\right] \\
& +(1-p)\left[p R_{1}\left(\tau_{1}+\tilde{X}_{1}, \tau_{2}+X_{1}, \cdots, \tau_{m} ; l-2, y_{2}+1 ; 2, i_{1}, i_{2}, \cdots, i_{k} ; \mathrm{P} 1\right)\right. \\
& \left.\left.+(1-p) R_{1}\left(\tau_{1}+\tilde{X}_{1}, \tau_{2}+X_{1}, \cdots, \tau_{m} ; l-2, y_{2} ; i_{1}, i_{2}, \cdots, i_{k} ; \mathrm{P} 1\right)\right]\right\} \\
= & \boldsymbol{E}\left\{\dot{g}_{1}\left(\tau_{1}+\tilde{X}_{1}\right)+p R_{1}\left(\tau_{1}+\tilde{X}_{1}, \tau_{2}, \cdots, \tau_{m} ; l-1, y_{2}+1 ; 1, i_{1}, i_{2}, \cdots, i_{k} ; \mathrm{P} 1\right)\right. \\
& \left.+(1-p) R_{1}\left(\tau_{1}+\tilde{X}_{1}, \tau_{2}, \cdots, \tau_{m} ; l-1, y_{2} ; i_{1}, i_{2}, \cdots, i_{k} ; \mathrm{P} 1\right)\right\},
\end{aligned}
$$

where $\tilde{X}_{1}$ is a random variable independent of and identically distributed to $X_{1}$.

Proof of Theorem 1. The proof is by induction on the total number of jobs. The result is trivially true (i.e. strategy $\mathrm{P} 1$ is optimal) when there is one job to be processed $\left(y_{1}=1\right.$ or $\left.y_{2}=1\right)$. We assume that it is true when $y_{1}+y_{2}=l-1$ and prove it for $y_{1}+y_{2}=l$. We also assume that there exists at least one job in each queue at the time the first server becomes available, because otherwise there is nothing to prove. Let S2 be a strategy that starts with a job in queue 2 . By the induction hypothesis it is optimal to follow P1 afterwards. We construct strategy S1 as follows: S1 starts with a job in queue 1, then processes a job in queue 2, and follows P1 afterwards. We need to show that

$$
\begin{aligned}
\Delta= & R\left(\tau_{1}, \tau_{2}, \cdots, \tau_{m} ; y_{1}, y_{2} ; i_{1}, i_{2}, \cdots, i_{k} ; \mathrm{S} 1\right) \\
& -R\left(\tau_{1}, \tau_{2}, \cdots, \tau_{m} ; y_{1}, y_{2} ; i_{1}, i_{2}, \cdots, i_{k} ; \mathrm{S} 2\right) \geqq 0 .
\end{aligned}
$$

After we prove that $\Delta \geqq 0$, repeated application of the same exchange argument will establish that P1 is the optimal policy, that is

$$
\begin{aligned}
& R\left(\tau_{1}, \tau_{2}, \cdots, \tau_{m} ; y_{1}, y_{2} ; i_{1}, i_{2}, \cdots, i_{k} ; \mathrm{P} 1\right) \\
& \quad \geqq R\left(\tau_{1}, \tau_{2}, \cdots, \tau_{m} ; y_{1}, y_{2} ; i_{1}, i_{2}, \cdots, i_{k} ; \mathrm{S}\right),
\end{aligned}
$$

for any policy S.

To show that $\Delta \geqq 0$ we proceed in two steps.

Step 1. We prove that

$$
\begin{aligned}
\Delta(\tau)= & \boldsymbol{E}\left\{r\left(\tau_{1}, \tau_{2}, \cdots, \tau_{m} ; y_{1}, y_{2} ; i_{1}, i_{2}, \cdots, i_{k} ; \mathrm{S} 1\right)\right. \\
& \left.-r\left(\tau_{1}, \tau_{2}, \cdots, \tau_{m} ; y_{1}, y_{2} ; i_{1}, i_{2}, \cdots, i_{k} ; \mathrm{S} 2\right) \mid X_{2}=\tau\right\}
\end{aligned}
$$

is non-decreasing in $\tau$. Assuming the result of Step 1 is true we have by $X_{2} \geqq_{\mathrm{st}} X_{1}$ 
and Proposition 8.1.2 of Ross (1983) that $\Delta=\boldsymbol{E}\left[\Delta\left(X_{2}\right)\right] \geqq \boldsymbol{E}\left[\Delta\left(\bar{X}_{1}\right)\right]$, where $\bar{X}_{1}$ is a random variable independent of $X_{1}, X_{2}$ and identically distributed to $X_{1}$.

Step 2. We prove that $\boldsymbol{E}\left[\Delta\left(\bar{X}_{1}\right)\right] \geqq 0$.

We proceed now to prove the claims made above.

Proof of Step 1. Without loss of generality we assume that $\tau_{1} \leqq \tau_{2} \leqq \cdots \leqq \tau_{m}$. We consider two cases.

Case 1. $\tau \geqq \tau_{2}-\tau_{1}$.

$$
\begin{aligned}
\Delta(\tau)= & \boldsymbol{E}\left\{1 ( X _ { 1 } < \tau _ { 2 } - \tau _ { 1 } ) \left[g_{1}\left(\tau_{1}+X_{1}\right)+g_{2}\left(\tau_{1}+X_{1}+\tau\right)\right.\right. \\
& +p R\left(\tau_{1}+X_{1}+\tau, \tau_{2}, \cdots, \tau_{m} ; y_{1}-1, y_{2} ; i_{1}, i_{2}, \cdots, i_{k} ; \mathrm{P} 1\right) \\
& \left.+(1-p) R\left(\tau_{1}+X_{1}+\tau, \tau_{2}, \cdots, \tau_{m} ; y_{1}-1, y_{2}-1 ; i_{1}, i_{2}, \cdots, i_{k} ; \mathrm{P} 1\right)\right] \\
& +1\left(X_{1} \geqq \tau_{2}-\tau_{1}\right)\left[g_{1}\left(\tau_{1}+X_{1}\right)+g_{2}\left(\tau_{2}+\tau\right)\right. \\
& +p R\left(\tau_{1}+X_{1}, \tau_{2}+\tau, \cdots, \tau_{m} ; y_{1}-1, y_{2} ; 1, i_{1}, i_{2}, \cdots, i_{k} ; \mathrm{P} 1\right) \\
& \left.+(1-p) R\left(\tau_{1}+X_{1}, \tau_{2}+\tau, \cdots, \tau_{m} ; y_{1}-1, y_{2}-1 ; i_{1}, i_{2}, \cdots, i_{k} ; \mathrm{P} 1\right)\right] \\
& -g_{2}\left(\tau_{1}+\tau\right)-g_{1}\left(\tau_{2}+X_{1}\right) \\
& -p R\left(\tau_{1}+\tau, \tau_{2}+X_{1}, \cdots, \tau_{m} ; y_{1}-1, y_{2} ; 2, i_{1}, i_{2}, \cdots, i_{k} ; \mathrm{P} 1\right) \\
& \left.-(1-p) R\left(\tau_{1}+\tau, \tau_{2}+X_{1}, \cdots, \tau_{m} ; y_{1}-1, y_{2}-1 ; i_{1}, i_{2}, \cdots, i_{k} ; \mathrm{P} 1\right)\right\}
\end{aligned}
$$

Differentiation of the above and Lemma 1 give

$$
\begin{aligned}
\frac{d \Delta(\tau)}{d \tau}= & \boldsymbol{E}\left\{1 ( X _ { 1 } < \tau _ { 2 } - \tau _ { 1 } ) \left[\dot{g}_{2}\left(\tau_{1}+X_{1}+\tau\right)\right.\right. \\
& +p R_{1}\left(\tau_{1}+X_{1}+\tau, \tau_{2}, \cdots, \tau_{m} ; y_{1}-1, y_{2} ; i_{1}, i_{2}, \cdots, i_{k} ; \mathrm{P} 1\right) \\
& \left.+(1-p) R_{1}\left(\tau_{1}+X_{1}+\tau, \tau_{2}, \cdots, \tau_{m} ; y_{1}-1, y_{2}-1 ; i_{1}, i_{2}, \cdots, i_{k} ; \mathrm{P} 1\right)\right] \\
& +1\left(X_{1} \geqq \tau_{2}-\tau_{1}\right)\left[\dot{g}_{2}\left(\tau_{2}+\tau\right)\right. \\
& +p R_{1}\left(\tau_{2}+\tau, \tau_{1}+X_{1}, \cdots, \tau_{m} ; y_{1}-1, y_{2} ; 2, i_{1}, i_{2}, \cdots, i_{k} ; \mathrm{P} 1\right) \\
& \left.+(1-p) R_{1}\left(\tau_{2}+\tau, \tau_{1}+X_{1}, \cdots, \tau_{m} ; y_{1}-1, y_{2}-1 ; i_{1}, i_{2}, \cdots, i_{k} ; \mathrm{P} 1\right)\right] \\
& -\dot{g}_{2}\left(\tau_{1}+\tau\right)-p R_{1}\left(\tau_{1}+\tau, \tau_{2}+X_{1}, \cdots, \tau_{m} ; y_{1}-1, y_{2} ; 2, i_{1}, i_{2}, \cdots, i_{k} ; \mathrm{P} 1\right) \\
& \left.-(1-p) R_{1}\left(\tau_{1}+\tau, \tau_{2}+X_{1}, \cdots, \tau_{m} ; y_{1}-1, y_{2}-1 ; i_{1}, i_{2}, \cdots, i_{k} ; \mathrm{P} 1\right)\right\} .
\end{aligned}
$$


Since $\tau_{1}+X_{1}+\tau \geqq \tau_{2}$ we have

$$
\begin{aligned}
R_{1}\left(\tau_{1}+X_{1}+\tau, \tau_{2}, \cdots, \tau_{m} ; y_{1}-1, y_{2} ; i_{1}, i_{2}, \cdots, i_{k} ; \mathrm{P} 1\right) \\
\quad=R_{1}\left(\tau_{1}+X_{1}+\tau, \tau_{2}, \cdots, \tau_{m} ; y_{1}-1, y_{2} ; 2, i_{1}, i_{2}, \cdots, i_{k} ; \mathrm{P} 1\right) .
\end{aligned}
$$

Having observed this we can apply Lemma 3 to get that the expression over which the expectation is taken is non-negative. Therefore $d \Delta(\tau) / d \tau \geqq 0$ for $\tau \geqq \tau_{2}-\tau_{1}$.

Case 2. $\tau<\tau_{2}-\tau_{1}$. Conditioning on the value of $X_{1}$ we get

$$
\begin{aligned}
\Delta(\tau)= & \boldsymbol{E}\left[\Delta(\tau) \mid X_{1}<\tau_{2}-\tau_{1}\right] \operatorname{Pr}\left(X_{1}<\tau_{2}-\tau_{1}\right) \\
& +\boldsymbol{E}\left[\Delta(\tau) \mid X_{1} \geqq \tau_{2}-\tau_{1}\right] \operatorname{Pr}\left(X_{1} \geqq \tau_{2}-\tau_{1}\right),
\end{aligned}
$$

where

$$
\begin{aligned}
& \boldsymbol{E}\left[\Delta(\tau) \mid X_{1}<\tau_{2}-\tau_{1}\right]=\boldsymbol{E}\left\{g_{1}\left(\tau_{1}+X_{1}\right)+g_{2}\left(\tau_{1}+X_{1}+\tau\right)\right. \\
& \quad+p R\left(\tau_{1}+X_{1}+\tau, \tau_{2}, \cdots, \tau_{m} ; y_{1}-1, y_{2} ; i_{1}, i_{2}, \cdots, i_{k} ; \mathrm{P} 1\right) \\
& +(1-p) R\left(\tau_{1}+X_{1}+\tau, \tau_{2}, \cdots, \tau_{m} ; y_{1}-1, y_{2}-1 ; i_{1}, i_{2}, \cdots, i_{k} ; \mathrm{P} 1\right) \\
& -g_{2}\left(\tau_{1}+\tau\right)-g_{1}\left(\tau_{1}+X_{1}+\tau\right) \\
& -p R\left(\tau_{1}+X_{1}+\tau, \tau_{2}, \cdots, \tau_{m} ; y_{1}-1, y_{2} ; 1, i_{1}, i_{2}, \cdots, i_{k} ; \mathrm{P} 1\right) \\
& -(1-p) R\left(\tau_{1}+X_{1}+\tau, \tau_{2}, \cdots, \tau_{m} ;\right. \\
& \left.\left.y_{1}-1, y_{2}-1 ; i_{1}, i_{2}, \cdots, i_{k} ; \mathrm{P} 1\right) \mid X_{1}<\tau_{2}-\tau_{1}\right\},
\end{aligned}
$$

and

$$
\begin{aligned}
\boldsymbol{E}\left[\Delta(\tau) \mid X_{1} \geqq\right. & \left.\tau_{2}-\tau_{1}\right]=\boldsymbol{E}\left\{R\left(\tau_{2}, \tau_{2}, \cdots, \tau_{m} ; y_{1}, y_{2} ; i_{1}, i_{2}, \cdots, i_{k} ; \mathrm{P} 1^{\prime}\right)\right. \\
& \left.-g_{2}\left(\tau_{1}+\tau\right)-R\left(\tau_{2}+\tau, \tau_{2}, \cdots, \tau_{m} ; y_{1}, y_{2}-1 ; i_{1}, i_{2}, \cdots, i_{k} ; \mathrm{P} 1^{\prime \prime}\right)\right\},
\end{aligned}
$$

where $\mathrm{P}^{\prime}$ is a strategy that assigns a job in queue 1 requiring service time $Z_{1}$ to server $1\left(Z_{1}\right.$ is the remaining service time for a job in queue 1 that has completed $\tau_{2}-\tau_{1}$ units of service), a job in queue 2 to server 2, and is identical to P1 afterwards, and $\mathrm{P} 1$ " is a strategy that assigns a job in queue 1 requiring service time $Z_{1}$ to server 1 , and is identical to $\mathrm{P} 1$ otherwise. Thus

$$
\begin{aligned}
\boldsymbol{E}[ & \left.\Delta(\tau) \mid X_{1} \geqq \tau_{2}-\tau_{1}\right]=\boldsymbol{E}\left\{g_{1}\left(\tau_{2}+Z_{1}\right)+g_{2}\left(\tau_{2}+\tau\right)\right. \\
& +p R\left(\tau_{2}+Z_{1}, \tau_{2}+\tau, \cdots, \tau_{m} ; y_{1}-1, y_{2} ; 1, i_{1}, i_{2}, \cdots, i_{k} ; \mathrm{P} 1\right) \\
& +(1-p) R\left(\tau_{2}+Z_{1}, \tau_{2}+\tau, \cdots, \tau_{m} ; y_{1}-1, y_{2}-1 ; i_{1}, i_{2}, \cdots, i_{k} ; \mathrm{P} 1\right) \\
& -g_{2}\left(\tau_{1}+\tau\right)-g_{1}\left(\tau_{2}+\tau+Z_{1}\right) \\
& -p R\left(\tau_{2}+\tau+Z_{1}, \tau_{2}, \cdots, \tau_{m} ; y_{1}-1, y_{2} ; 1, i_{1}, i_{2}, \cdots, i_{k} ; \mathrm{P} 1\right) \\
& \left.-(1-p) R\left(\tau_{2}+\tau+Z_{1}, \tau_{2}, \cdots, \tau_{m} ; y_{1}-1, y_{2}-1 ; i_{1}, i_{2}, \cdots, i_{k} ; \mathrm{P} 1\right)\right\} .
\end{aligned}
$$


From Equations (2.11)-(2.13) and Lemma 1 we get

$$
\begin{aligned}
\frac{d \Delta(\tau)}{d \tau}= & \operatorname{Pr}\left(X_{1}<\tau_{2}-\tau_{1}\right) \boldsymbol{E}\left\{\dot{g}_{2}\left(\tau_{1}+X_{1}+\tau\right)\right. \\
& +p R_{1}\left(\tau_{1}+X_{1}+\tau, \tau_{2}, \cdots, \tau_{m} ; y_{1}-1, y_{2} ; i_{1}, i_{2}, \cdots, i_{k} ; \mathrm{P} 1\right) \\
& +(1-p) R_{1}\left(\tau_{1}+X_{1}+\tau, \tau_{2}, \cdots, \tau_{m} ; y_{1}-1, y_{2}-1 ; i_{1}, i_{2}, \cdots, i_{k} ; \mathrm{P} 1\right) \\
& -\dot{g}_{2}\left(\tau_{1}+\tau\right)-\dot{g}_{1}\left(\tau_{1}+X_{1}+\tau\right) \\
& -p R_{1}\left(\tau_{1}+X_{1}+\tau, \tau_{2}, \cdots, \tau_{m} ; y_{1}-1, y_{2} ; 1, i_{1}, i_{2}, \cdots, i_{k} ; \mathrm{P} 1\right)-(1-p) \\
& \left.\times R_{1}\left(\tau_{1}+X_{1}+\tau, \tau_{2}, \cdots, \tau_{m} ; y_{1}-1, y_{2}-1 ; i_{1}, i_{2}, \cdots, i_{k} ; \mathrm{P} 1\right) \mid X_{1}<\tau_{2}-\tau_{1}\right\} \\
& +\operatorname{Pr}\left(X_{1} \geqq \tau_{2}-\tau_{1}\right) \boldsymbol{E}\left\{\dot{g}_{2}\left(\tau_{2}+\tau\right)\right. \\
& +p R_{1}\left(\tau_{2}+\tau, \tau_{2}+Z_{1}, \cdots, \tau_{m} ; y_{1}-1, y_{2} ; 2, i_{1}, i_{2}, \cdots, i_{k} ; \mathrm{P} 1\right) \\
& +(1-p) R_{1}\left(\tau_{2}+\tau, \tau_{2}+Z_{1}, \cdots, \tau_{m} ; y_{1}-1, y_{2}-1 ; i_{1}, i_{2}, \cdots, i_{k} ; \mathrm{P} 1\right) \\
& -\dot{g}_{2}\left(\tau_{1}+\tau\right)-\dot{g}_{1}\left(\tau_{2}+\tau+Z_{1}\right) \\
& -p R_{1}\left(\tau_{2}+\tau+Z_{1}, \tau_{2}, \cdots, \tau_{m} ; y_{1}-1, y_{2} ; 1, i_{1}, i_{2}, \cdots, i_{k} ; \mathrm{P} 1\right) \\
& \left.-(1-p) R_{1}\left(\tau_{2}+\tau+Z_{1}, \tau_{2}, \cdots, \tau_{m} ; y_{1}-1, y_{2}-1 ; i_{1}, i_{2}, \cdots, i_{k} ; \mathrm{P} 1\right)\right\} .
\end{aligned}
$$

The expression over which the first expectation is taken is non-negative because of Lemma $2 \mathrm{a}$ and $\dot{g}_{2}\left(\tau_{1}+\tau\right)$ being negative. Since $X_{1}$ is a random variable that has non-decreasing likelihood ratio, $Z_{1} \leqq_{\text {st }} X_{1}$. Thus, according to Lemma 3 , replacing $Z_{1}$ by $X_{1}$ in Equation (2.14) we obtain

$$
\begin{aligned}
\frac{d \Delta(\tau)}{d \tau} \geqq & \operatorname{Pr}\left(X_{1} \geqq \tau_{2}-\tau_{1}\right) \boldsymbol{E}\left\{\dot{g}_{2}\left(\tau_{2}+\tau\right)\right. \\
& +p R_{1}\left(\tau_{2}+\tau, \tau_{2}+X_{1}, \cdots, \tau_{m} ; y_{1}-1, y_{2} ; 2, i_{1}, i_{2}, \cdots, i_{k} ; \mathrm{P} 1\right) \\
& +(1-p) R_{1}\left(\tau_{2}+\tau, \tau_{2}+X_{1}, \cdots, \tau_{m} ; y_{1}-1, y_{2}-1 ; i_{1}, i_{2}, \cdots, i_{k} ; \mathrm{P} 1\right) \\
& -\dot{g}_{2}\left(\tau_{1}+\tau\right)-\dot{g}_{1}\left(\tau_{2}+\tau+X_{1}\right) \\
& -p R_{1}\left(\tau_{2}+\tau+X_{1}, \tau_{2}, \cdots, \tau_{m} ; y_{1}-1, y_{2} ; 1, i_{1}, i_{2}, \cdots, i_{k} ; \mathrm{P} 1\right) \\
& \left.-(1-p) R_{1}\left(\tau_{2}+\tau+X_{1}, \tau_{2}, \cdots, \tau_{m} ; y_{1}-1, y_{2}-1 ; i_{1}, i_{2}, \cdots, i_{k} ; \mathrm{P} 1\right)\right\} \\
\geqq & \operatorname{Pr}\left(X_{1} \geqq \tau_{2}-\tau_{1}\right) \boldsymbol{E}\left\{\dot{g}_{2}\left(\tau_{2}+\tau\right)\right. \\
& +p R_{1}\left(\tau_{2}+\tau, \tau_{2}+X_{1}, \cdots, \tau_{m} ; y_{1}-1, y_{2} ; 2, i_{1}, i_{2}, \cdots, i_{k} ; \mathrm{P} 1\right) \\
& +(1-p) R_{1}\left(\tau_{2}+\tau, \tau_{2}+X_{1}, \cdots, \tau_{m} ; y_{1}-1, y_{2}-1 ; i_{1}, i_{2}, \cdots, i_{k} ; \mathrm{P} 1\right) \\
& -\dot{g}_{2}\left(\tau_{1}+\tau\right) \\
& \left.-R_{1}\left(\tau_{2}+\tau, \tau_{2}, \cdots, \tau_{m} ; y_{1}, y_{2}-1 ; i_{1}, i_{2}, \cdots, i_{k} ; \mathrm{P} 1\right)\right\}
\end{aligned}
$$


where the second inequality follows from Lemma $4 \mathrm{~b}$. We have

$$
\begin{aligned}
& R_{1}\left(\tau_{2}+\tau, \tau_{2}, \cdots, \tau_{m} ; y_{1}, y_{2}-1 ; i_{1}, i_{2}, \cdots, i_{k} ; \mathrm{P} 1\right) \\
&= \boldsymbol{E}\left\{p R_{1}\left(\tau_{2}+\tau, \tau_{2}+X_{1}, \cdots, \tau_{m} ; y_{1}-1, y_{2} ; 2, i_{1}, i_{2}, \cdots, i_{k} ; \mathrm{P} 1\right)\right. \\
&\left.+(1-p) R_{1}\left(\tau_{2}+\tau, \tau_{2}+X_{1}, \cdots, \tau_{m} ; y_{1}-1, y_{2}-1 ; i_{1}, i_{2}, \cdots, i_{k} ; \mathrm{P} 1\right)\right\} .
\end{aligned}
$$

Therefore, after substitution of (2.16) into (2.15) we obtain

$$
\frac{d \Delta(\tau)}{d \tau} \geqq \operatorname{Pr}\left(X_{1} \geqq \tau_{2}-\tau_{1}\right)\left[\dot{g}_{2}\left(\tau_{2}+\tau\right)-\dot{g}_{2}\left(\tau_{1}+\tau\right)\right] \geqq 0,
$$

and the proof of Step 1 is complete.

Proof of Step 2.

$$
\begin{aligned}
\boldsymbol{E} & \left.\Delta\left(\bar{X}_{1}\right)\right]=\boldsymbol{E}\left\{g_{1}\left(\tau_{1}+X_{1}\right)+g_{2}\left(\tau_{1}+X_{1}+\bar{X}_{1}\right)\right. \\
& +p R\left(\tau_{1}+X_{1}+\bar{X}_{1}, \tau_{2}, \cdots, \tau_{m} ; y_{1}-1, y_{2} ; i_{1}, i_{2}, \cdots, i_{k} ; \mathrm{P} 1\right)+(1-p) \\
& \left.\times R\left(\tau_{1}+X_{1}+\bar{X}_{1}, \tau_{2}, \cdots, \tau_{m} ; y_{1}-1, y_{2}-1 ; i_{1}, i_{2}, \cdots, i_{k} ; \mathrm{P} 1\right) \mid X_{1}<\tau_{2}-\tau_{1}\right\} \\
& \times \operatorname{Pr}\left(X_{1}<\tau_{2}-\tau_{1}\right)+\boldsymbol{E}\left\{g_{1}\left(\tau_{2}+Z_{1}\right)+g_{2}\left(\tau_{2}+\bar{X}_{1}\right)\right. \\
& +p R\left(\tau_{2}+Z_{1}, \tau_{2}+\bar{X}_{1}, \cdots, \tau_{m} ; y_{1}-1, y_{2} ; 1, i_{1}, i_{2}, \cdots, i_{k} ; \mathrm{P} 1\right) \\
& \left.+(1-p) R\left(\tau_{2}+Z_{1}, \tau_{2}+\bar{X}_{1}, \cdots, \tau_{m} ; y_{1}-1, y_{2}-1 ; i_{1}, i_{2}, \cdots, i_{k} ; \mathrm{P} 1\right)\right\} \\
& \times \operatorname{Pr}\left(X_{1} \geqq \tau_{2}-\tau_{1}\right)-\boldsymbol{E}\left\{g_{2}\left(\tau_{1}+\bar{X}_{1}\right)+g_{1}\left(\tau_{1}+\bar{X}_{1}+X_{1}\right)\right. \\
& +p R\left(\tau_{1}+\bar{X}_{1}+X_{1}, \tau_{2}, \cdots, \tau_{m} ; y_{1}-1, y_{2} ; 1, i_{1}, i_{2}, \cdots, i_{k} ; \mathrm{P} 1\right)+(1-p) \\
& \left.\times R\left(\tau_{1}+\bar{X}_{1}+X_{1}, \tau_{2}, \cdots, \tau_{m} ; y_{1}-1, y_{2}-1 ; i_{1}, i_{2}, \cdots, i_{k} ; \mathrm{P} 1\right) \mid \bar{X}_{1}<\tau_{2}-\tau_{1}\right\} \\
& \times \operatorname{Pr}\left(\bar{X}_{1}<\tau_{2}-\tau_{1}\right)-\boldsymbol{E}\left\{g_{2}\left(\tau_{2}+\bar{Z}_{1}\right)+g_{1}\left(\tau_{2}+X_{1}\right)\right. \\
& +p R\left(\tau_{2}+\bar{Z}_{1}, \tau_{2}+X_{1}, \cdots, \tau_{m} ; y_{1}-1, y_{2} ; 2, i_{1}, i_{2}, \cdots, i_{k} ; \mathrm{P} 1\right) \\
& \left.+(1-p) R\left(\tau_{2}+\bar{Z}_{1}, \tau_{2}+X_{1}, \cdots, \tau_{m} ; y_{1}-1, y_{2}-1 ; i_{1}, i_{2}, \cdots, i_{k} ; \mathrm{P} 1\right)\right\} \\
& \times \operatorname{Pr}\left(\bar{X}_{1} \geqq \tau_{2}-\tau_{1}\right),
\end{aligned}
$$

where $Z_{1}, \bar{Z}_{1}$ are the remaining service times of jobs having service times $X_{1}, \bar{X}_{1}$ after completing $\tau_{2}-\tau_{1}$ units of service. Note that $Z_{1}, \bar{Z}_{1}$ are independent and identically distributed, and $Z_{1} \leqslant_{\mathrm{st}} X_{1}$, because $X_{1}$ has non-decreasing likelihood 
ratio.

$$
\begin{aligned}
& \boldsymbol{E}\left\{g_{1}\left(\tau_{1}+X_{1}\right)+g_{2}\left(\tau_{1}+X_{1}+\bar{X}_{1}\right) \mid X_{1}<\tau_{2}-\tau_{1}\right\} \\
& \quad-\boldsymbol{E}\left\{g_{2}\left(\tau_{1}+\bar{X}_{1}\right)+g_{1}\left(\tau_{1}+\bar{X}_{1}+X_{1}\right) \mid \bar{X}_{1}<\tau_{2}-\tau_{1}\right\} \\
&=\quad \boldsymbol{E}\left\{\left(C_{1}-p C_{2}\right) \exp \left(-\alpha\left(\tau_{1}+X_{1}\right)\right)\right. \\
&\left.\quad+C_{2} \exp \left(-\alpha\left(\tau_{1}+X_{1}+\bar{X}_{1}\right)\right) \mid X_{1}<\tau_{2}-\tau_{1}\right\} \\
& \quad-\boldsymbol{E}\left\{C_{2} \exp \left(-\alpha\left(\tau_{1}+X_{1}\right)\right)\right. \\
&\left.\quad+\left(C_{1}-p C_{2}\right) \exp \left(-\alpha\left(\tau_{1}+X_{1}+\bar{X}_{1}\right)\right) \mid X_{1}<\tau_{2}-\tau_{1}\right\} \\
&= \boldsymbol{E}\left\{[ ( C _ { 1 } - p C _ { 2 } ) - C _ { 2 } ] \left(\exp \left(-\alpha\left(\tau_{1}+X_{1}\right)\right)\right.\right. \\
&\left.\left.-\exp \left(-\alpha\left(\tau_{1}+X_{1}+\bar{X}_{1}\right)\right)\right) \mid X_{1}<\tau_{2}-\tau_{1}\right\} \geqq 0,
\end{aligned}
$$

because of condition (iii) in the theorem.

$$
\begin{aligned}
& \boldsymbol{E}\left\{g_{1}\left(\tau_{2}+Z_{1}\right)+g_{2}\left(\tau_{2}+\bar{X}_{1}\right)\right\}-\boldsymbol{E}\left\{g_{2}\left(\tau_{2}+\bar{Z}_{1}\right)+g_{1}\left(\tau_{2}+X_{1}\right)\right\} \\
&= \boldsymbol{E}\left\{\left(C_{1}-p C_{2}\right) \exp \left(-\alpha\left(\tau_{2}+Z_{1}\right)\right)+C_{2} \exp \left(-\alpha\left(\tau_{2}+X_{1}\right)\right)\right\} \\
&-\boldsymbol{E}\left\{C_{2} \exp \left(-\alpha\left(\tau_{2}+Z_{1}\right)\right)+\left(C_{1}-p C_{2}\right) \exp \left(-\alpha\left(\tau_{2}+X_{1}\right)\right)\right\} \\
&= \boldsymbol{E}\left\{\left[\left(C_{1}-p C_{2}\right)-C_{2}\right]\left(\exp \left(-\alpha\left(\tau_{2}+Z_{1}\right)\right)-\exp \left(-\alpha\left(\tau_{2}+X_{1}\right)\right)\right)\right\} \geqq 0,
\end{aligned}
$$

because of condition (iii) in the theorem and $Z_{1} \leqq{ }_{\text {st }} X_{1}$.

We can show by a sample path argument that

$$
\begin{aligned}
& \boldsymbol{E}\left\{R\left(\tau_{1}+X_{1}+\bar{X}_{1}, \tau_{2}, \cdots, \tau_{m} ; y_{1}-1, y_{2} ; i_{1}, i_{2}, \cdots, i_{k} ; \mathrm{P} 1\right) \mid X_{1}<\tau_{2}-\tau_{1}\right\} \\
& \geqq \boldsymbol{E}\left\{R\left(\tau_{1}+\bar{X}_{1}+X_{1}, \tau_{2}, \cdots, \tau_{m} ; y_{1}-1, y_{2} ; 1, i_{1}, i_{2}, \cdots, i_{k} ; \mathrm{P} 1\right) \mid \bar{X}_{1}<\tau_{2}-\tau_{1}\right\} .
\end{aligned}
$$

This is true for the following reason. The conditional distributions of $\tau_{1}+X_{1}+\bar{X}_{1}$ given $X_{1}<\tau_{2}-\tau_{1}$ and $\tau_{1}+\bar{X}_{1}+X_{1}$ given $\bar{X}_{1}<\tau_{2}-\tau_{1}$ are identical. We observe that the right-hand side and left-hand side of (2.20) have the same number of jobs in both queues. However, when considering the right-hand side we note that one of the jobs in queue 2 results from an interconnection at server 1 . Consequently, the corresponding job on the left-hand side of (2.20) may be available at an earlier time, thus, yielding a larger reward under policy P1. Using a similar sample path argument we can show that, for $x \geqq y$,

$$
\begin{aligned}
& R\left(y, x, \cdots, \tau_{m} ; y_{1}-1, y_{2} ; 1, i_{1}, i_{2}, \cdots, i_{k} ; \mathrm{P} 1\right) \\
& \quad \geqq R\left(y, x, \cdots, \tau_{m} ; y_{1}-1, y_{2} ; 2, i_{1}, i_{2}, \cdots, i_{k} ; \mathrm{P} 1\right) .
\end{aligned}
$$

(The reason for (2.21) is the following. Considering the right-hand side of (2.21), one of the jobs in queue 2 results from an interconnection at server 2, whereas the corresponding job in the left-hand side of (2.21) results from an interconnection at server 1 at an earlier time.) 
Moreover,

$$
\begin{aligned}
& R\left(y, x, \cdots, \tau_{m} ; y_{1}-1, y_{2} ; 2, i_{1}, i_{2}, \cdots, i_{k} ; \mathrm{P} 1\right) \\
& \quad=R\left(x, y, \cdots, \tau_{m} ; y_{1}-1, y_{2} ; 1, i_{1}, i_{2}, \cdots, i_{k} ; \mathrm{P} 1\right) .
\end{aligned}
$$

Let $Z, X$ be independent random variables identically distributed to $\tau_{2}+Z_{1}$, $\tau_{2}+X_{1}$ respectively. Since $X_{1}$ has non-decreasing likelihood ratio, we have $Z \leqq_{\mathrm{LR}} X$. From (2.21), (2.22) and Proposition 8.4.2 of Ross (1983) we get

$$
\begin{gathered}
R\left(Z, X, \cdots, \tau_{m} ; y_{1}-1, y_{2} ; 1, i_{1}, i_{2}, \cdots, i_{k} ; \mathrm{P} 1\right) \\
\geqq_{\mathrm{st}} R\left(X, Z, \cdots, \tau_{m} ; y_{1}-1, y_{2} ; 1, i_{1}, i_{2}, \cdots, i_{k} ; \mathrm{P} 1\right) \\
\quad=R\left(Z, X, \cdots, \tau_{m} ; y_{1}-1, y_{2} ; 2, i_{1}, i_{2}, \cdots, i_{k} ; \mathrm{P} 1\right) .
\end{gathered}
$$

Consequently, by Lemma 8.1 .1 of Ross (1983) we get

$$
\begin{aligned}
\boldsymbol{E} & \left\{R\left(\tau_{2}+Z_{1}, \tau_{2}+\bar{X}_{1}, \cdots, \tau_{m} ; y_{1}-1, y_{2} ; 1, i_{1}, i_{2}, \cdots, i_{k} ; \mathrm{P} 1\right)\right\} \\
& =\boldsymbol{E}\left\{R\left(Z, X, \cdots, \tau_{m} ; y_{1}-1, y_{2} ; 1, i_{1}, i_{2}, \cdots, i_{k} ; \mathrm{P} 1\right)\right\} \\
& \geqq \boldsymbol{E}\left\{R\left(Z, X, \cdots, \tau_{m} ; y_{1}-1, y_{2} ; 2, i_{1}, i_{2}, \cdots, i_{k} ; \mathrm{P} 1\right)\right\} \\
& =\boldsymbol{E}\left\{R\left(\tau_{2}+\bar{Z}_{1}, \tau_{2}+X_{1}, \cdots, \tau_{m} ; y_{1}-1, y_{2} ; 2, i_{1}, i_{2}, \cdots, i_{k} ; \mathrm{P} 1\right)\right\} .
\end{aligned}
$$

From Equations (2.17), (2.18), (2.19), (2.20), and (2.23) we get $\boldsymbol{E}\left[\Delta\left(\bar{X}_{1}\right)\right] \geqq 0$, and the proof of Step 2 and the theorem is complete.

\section{Discussion}

We have considered multiserver scheduling in a system of two interconnected queues with jobs completing service in queue 1 joining queue 2 with a certain probability. We have shown that when jobs in queue 1 require a stochastically smaller service time and yield a higher reward than jobs in queue 2 (conditions (i) and (iii) of the theorem), the strategy that gives priority to queue 1 maximizes the expected reward. In this section we present counterexamples showing that if any of the sufficient conditions under which strategy P1 is optimal is relaxed, the optimality of P1 is no longer guaranteed. We also present a counterexample showing that policy $\mathrm{P} 1$ is not optimal when conditions similar to (i)-(iii) of Theorem 1 are true but the queues are interconnected in the opposite way, i.e. jobs completing service in queue 2 (the queue with the smallest expected reward and stochastically larger service rate) join queue 1 with a certain probability. Finally, by example we illustrate the following points: (i) the conditions of Theorem 1 are sufficient but not necessary; and (ii) the index that describes the optimal policy in the single-server problem is suboptimal in the multiserver problem. 
We first present counterexamples showing that strategy P1 is not necessarily optimal when one of conditions (i) and (iii) in the theorem is not satisfied. Jobs from queue 1 join queue 2 with probability 1 . Service times are exponential with rates $\mu_{1}$, $\mu_{2}$. We take $y_{1}=1, y_{2}=2$, and two servers $(m=2)$. Strategy P1 initially assigns one server to each queue, whereas strategy P2 assigns both servers to queue 2. A straightforward but tedious calculation shows that the expected rewards under strategies P1 and P2 are

$$
\begin{aligned}
R(\mathrm{P} 1)= & \left(C_{1}-C_{2}\right) \frac{\mu_{1}}{\alpha+\mu_{1}}+C_{2}\left[\frac{\mu_{1} \mu_{2}}{\left(\alpha+\mu_{2}\right)\left(\alpha+\mu_{1}\right)}-\frac{\mu_{1} \mu_{2}}{\left(\alpha+2 \mu_{2}\right)\left(\alpha+\mu_{1}+\mu_{2}\right)}\right. \\
& +\frac{\mu_{2}\left(\alpha+2 \mu_{2}\right)}{\left(\alpha+\mu_{2}\right)^{2}}-\frac{\mu_{1} \mu_{2}^{2}}{\left(\alpha+\mu_{2}\right)^{2}\left(\alpha+\mu_{1}+\mu_{2}\right)}+\frac{\mu_{1} \mu_{2}}{\left(\alpha+\mu_{2}\right)\left(\alpha+\mu_{1}+\mu_{2}\right)} \\
& \left.+\frac{\mu_{1} \mu_{2}^{2}}{\left(\alpha+\mu_{2}\right)\left(\alpha+2 \mu_{2}\right)\left(\alpha+\mu_{1}+\mu_{2}\right)}\right], \\
R(\mathrm{P} 2)= & \left(C_{1}-C_{2}\right) \frac{2 \mu_{1} \mu_{2}}{\left(\alpha+\mu_{1}\right)\left(\alpha+2 \mu_{2}\right)} \\
& +C_{2}\left[\frac{2 \mu_{2}}{\alpha+2 \mu_{2}}+\frac{2 \mu_{1} \mu_{2}^{2}}{\left(\alpha+\mu_{2}\right)\left(\alpha+\mu_{1}\right)\left(\alpha+2 \mu_{2}\right)}+\frac{2 \mu_{2}^{2}}{\left(\alpha+\mu_{2}\right)\left(\alpha+2 \mu_{2}\right)}\right] .
\end{aligned}
$$

Counterexample 1. The condition on the service times is not satisfied. We take $\mu_{1}=1, \mu_{2}=2, C_{1}-C_{2}=C_{2}=1$, and $\alpha=1$. Substituting these values into (3.1) and (3.2) we get $R(\mathrm{P} 1)=2-\frac{1}{30}, R(\mathrm{P} 2)=2$, hence strategy $\mathrm{P} 2$ does better than $\mathrm{P} 1$.

Counterexample 2. The condition on the rewards is not satisfied. We take $\mu_{1}=\mu_{2}=\alpha=1, C_{1}-C_{2}=1$, and $C_{2}=2$. Substituting these values into (3.1) and (3.2) we get $R(\mathrm{P} 1)=\frac{23}{9}, R(\mathrm{P} 2)=\frac{24}{9}$, hence strategy $\mathrm{P} 2$ does better than $\mathrm{P} 1$.

We now give a counterexample to show that when the interconnection is from queue 2 to queue 1 , and jobs in queue 1 require a stochastically smaller service time and yield a higher reward $\left(g_{1}(t) \geqq g_{2}(t)\right.$ for all $\left.t\right)$ than jobs in queue 2 , the strategy that gives priority to queue 1 need not be optimal. Jobs from queue 2 join queue 1 with probability 1 . We take service times to be exponential with rates $\mu_{1}, \mu_{2}, y_{1}=2$, $y_{2}=1$, and two servers $(m=2)$. Note that the expected rewards under strategies P1 and $\mathrm{P} 2$ are given by (3.2) and (3.1) respectively with $C_{1}-C_{2}$ replaced by $C_{2}-C_{1}$, $C_{2}$ replaced by $C_{1}, \mu_{1}$ replaced by $\mu_{2}$, and $\mu_{2}$ replaced by $\mu_{1}$. Taking $\mu_{1}=\mu_{2}=\alpha=1, C_{2}-C_{1}=1$, and $C_{1}=1 \cdot 1$ we get $R(\mathrm{P} 1)=1 \cdot 616, R(\mathrm{P} 2)=1 \cdot 63$, hence strategy $\mathrm{P} 2$ does better than $\mathrm{P} 1$.

Next we present an example illustrating the following points: (i) the conditions of Theorem 1 are sufficient but not necessary for P1 to be optimal; (ii) the optimal policy for the single-server problem is not necessarily optimal for the multiserver 
problem. Consider the example given at the beginning of this section. Take $\mu_{1}=2$, $\mu_{2}=1, p=1, C_{1}-C_{2}=0.99, C_{2}=2$ and $\alpha=1$. Define

$$
\begin{aligned}
& v_{1}=\frac{\left(C_{1}-p C_{2}\right) \boldsymbol{E}\left(\exp \left(-\alpha X_{1}\right)\right)}{1-\boldsymbol{E}\left(\exp \left(-\alpha X_{1}\right)\right)}, \\
& \boldsymbol{v}_{2}=\frac{C_{2} \boldsymbol{E}\left(\exp \left(-\alpha X_{2}\right)\right)}{1-\boldsymbol{E}\left(\exp \left(-\alpha X_{2}\right)\right)}
\end{aligned}
$$

for exponential service times with rates $\mu_{1}, \mu_{2}$,

$$
\begin{aligned}
& v_{1}=\left(C_{1}-p C_{2}\right) \mu_{1}, \\
& v_{2}=C_{2} \mu_{2} .
\end{aligned}
$$

Note the following facts: (1) the optimal policy for the single-server problem is described by the following rule: priority is given to queue 1 if $v_{1} \geqq v_{2}$ and to queue 2 otherwise; (2) the conditions of Theorem 1 are not satisfied $\left(C_{1}-C_{2}<C_{2}\right)$. For this example the rewards for policies $\mathrm{P} 1$ and $\mathrm{P} 2$ are $R(\mathrm{P} 1)=2 \cdot 91$ and $R(\mathrm{P} 2)=2 \cdot 88$. For the single-server problem policy $\mathrm{P} 2$ is optimal since $C_{2} \mu_{2}=2>1.98=\left(C_{1}-C_{2}\right) \mu_{1}$.

Finally consider $N$ queues interconnected in the following way. Jobs that complete service in queue $i, i=1,2, \cdots, N-1$, join queue $j, j>i$, with probability $p_{i j}$, and leave the system with probability $1-\sum_{j>i} p_{i j}$. Jobs that complete service in queue $N$ leave the system. Consider a cost criterion similar to (1.1). We conjecture that if $X_{1} \leqq_{\mathrm{st}} X_{2} \leqq_{\mathrm{st}} \cdots \leqq_{\mathrm{st}} X_{N}, X_{1}, X_{2}, \cdots, X_{N-1}$ have non-decreasing likelihood ratio and

$$
\left(C_{1}-\sum_{j>1} p_{1 j} C_{j}\right) \geqq\left(C_{2}-\sum_{j>2} p_{2 j} C_{j}\right) \geqq \cdots \geqq C_{N},
$$

the optimal strategy serves the queues according to the priority list $1,2, \cdots, N$.

\section{Acknowledgement}

This work was supported in part by NSF Grant No. NSF-NCR-9204419.

\section{References}

Agrawal, R., Hegde, M. and Teneketzis, D. (1990) Multi-armed bandit problems with multiple plays and switching costs. Stoch. Stoch. Rep. 29, 437-459.

Bruno, J., Downey, P. And Frederickson, G. N. (1981) Sequencing tasks with exponential service times to minimize the expected flowtime or makespan. J. Assoc. Comp. Mach. 28, 100-113.

Chang, C. S., Nelson, R. AND Pinedo, M. (1991) Scheduling two classes of exponential jobs on parallel processors: structural results and worst-case analysis. Adv. Appl. Prob. 23, 925-944.

Coffman, E. G., Flatto, L., Garey, M. R. and Weber, R. R. (1987) Minimizing expected makespans on uniform processor systems. Adv. Appl. Prob. 19, 177-201.

GlazeBrooK, K. D. (1979) Scheduling tasks with exponential service times on parallel processors. $J$. Appl. Prob. 16, 685-689.

Glazebrook, K. D. ANd Nash, P. (1976) On multi-server stochastic scheduling. J. R. Statist. Soc. B 38, 67-72. 
KAMPKE, T. (1987) On the optimality of static priority policies in stochastic scheduling on parallel machines. J. Appl. Prob. 24, 430-448.

KAMPKE, T. (1989) Optimal scheduling of jobs with exponential service times on identical parallel processors. Operat. Res. 37, 126-133.

Lehtonen, T. (1988) Scheduling jobs with exponential processing times on parallel machines. J. Appl. Prob. 25, 752-762.

Ross S. (1983a) Introduction to Stochastic Dynamic Programming. Academic Press, New York.

Ross, S. (1983b) Stochastic Processes. Wiley, New York.

VAN Der Heyden, L. (1981) Scheduling jobs with exponential processing and arrival times on identical processors so as to minimize the expected makespan. Math. Operat. Res. 6, 305-312.

Weber, R. R. (1980) Optimal Organization of Multi-server Systems. Ph.D. Thesis, University of Cambridge.

WEBER, R. R. (1982) Scheduling jobs with stochastic processing requirements on parallel machines to minimize makespan or flowtime. J. Appl. Prob. 19, 167-182.

Weber, R. R. AND Weiss, G. (1990) On an index policy for restless bandits. J. Appl. Prob. 27, 637-648.

Weber, R. R., VaraiYa, P. AND WalRand, J. (1986) Scheduling jobs with stochastically ordered processing times on parallel machines to minimize expected flowtime. J. Appl. Prob. 23, 841-847.

WeIss, G. (1982) Multiserver stochastic scheduling. In Deterministic and Stochastic Scheduling, ed. M. A. Dempster and J. K. Lenstra, pp. 157-179. Kluwer, Dordrecht.

Weiss, G. AND PINEDO, M. (1980) Scheduling tasks with exponential service times on non-identical processors to minimize various cost functions. J. Appl. Prob. 17, 187-202.

WhitTle, P. (1988) Restless bandits: activity allocation in a changing world. In A Celebration of Applied Probability, ed. J. Gani, J. Appl. Prob. 25A, 287-298.

Xu, S. H., Mirchandani, P. B., Kumar, S. P. R. and Weber, R. R. (1990) Stochastic dispatching of multi-priority jobs to heterogeneous processors. J. Appl. Prob. 27, 852-861. 